\title{
NONEQUILIBRIUM DIFFUSIVE GAS DYNAMICS: POISEUILLE MICROFLOW
}

\author{
RAFAIL V. ABRAMOV AND JASMINE T. OTTO
}

\begin{abstract}
AвSTRACT. We test the recently developed hierarchy of diffusive moment closures for gas dynamics together with the near-wall viscosity scaling on the Poiseuille flow of argon and nitrogen in a one micrometer wide channel, and compare it against the corresponding Direct Simulation Monte Carlo computations. We find that the diffusive regularized Grad equations with viscosity scaling provide the most accurate approximation to the benchmark DSMC results. At the same time, the conventional Navier-Stokes equations without the near-wall viscosity scaling are found to be the least accurate among the tested closures.
\end{abstract}

\section{INTRODUCTION}

The diffusive Boltzmann equation and the corresponding hierarchy of the diffusive moment closure equations were derived in a recent work [1], including the equilibrium (diffusive Navier-Stokes) and nonequilibrium (diffusive Grad) closures. The additional mass-diffusive term originated from the difference between the deterministic real gas dynamics and the artificial "random gas" multimolecular process, which led to the conventional Boltzmann equation. Additionally, a near-wall viscosity scaling was computed in [2], based on the shortened mean free path of a gas molecule near a wall. The new equations were studied in a Couette microflow setting for argon and nitrogen, and it was found that the diffusive Navier-Stokes and Grad moment closures with the near-wall viscosity scaling developed Knudsen velocity boundary layers near the walls, closely matching the results of the corresponding DSMC computations. It was also found that the component of the heat flux parallel to the flow, produced by the DSMC computations, was captured quite well by the diffusive regularized Grad equations, but not by the conventional or diffusive Navier-Stokes closures.

In the present work, we test the developed hierarchy of diffusive moment closures [1] together with the near-wall viscosity scaling [2] on the Poiseuille flow of argon and nitrogen in a one micrometer wide channel, and compare it against the corresponding Direct Simulation Monte Carlo (DSMC) computations [4,11]. We find that the diffusive regularized Grad equations with viscosity scaling provide the most accurate approximation to the benchmark DSMC results. At the same time, the conventional Navier-Stokes equations are found to be the least accurate among the tested closures.

The paper is organized as follows. In Section 2 we present the diffusive closure equations [1] and near-wall viscosity scaling [2]. In Section 3 we show the results of the

Department of Mathematics, Statistics and Computer Science, University of Illinois at Chicago, 851 S. Morgan ST., Chicago, IL 60607

E-mail addresses: abramov@uic.edu, jotto3@uic.edu.

Date: September 25, 2018.

Key words and phrases. Diffusive fluid dynamics; Grad equations; Poiseuille flow. 
computational experiments with the Poiseuille flow. In Section 4 we summarize the results of the work.

\section{The Diffusive CLOSURES FOR CONTINUUM GAS DYNAMicS}

The diffusive equations for the density $\rho$, velocity $\boldsymbol{u}$ and energy $E$ are given by

$$
\begin{gathered}
\frac{\partial \rho}{\partial t}+\operatorname{div}(\rho \boldsymbol{u})=\operatorname{div}\left(\frac{D_{\alpha}}{p} \nabla p\right), \\
\frac{\partial(\rho \boldsymbol{u})}{\partial t}+\operatorname{div}\left(\rho\left(\boldsymbol{u} \boldsymbol{u}^{T}+\boldsymbol{T}\right)\right)=\operatorname{div}\left(\frac{D_{\alpha}}{p} \nabla(p \boldsymbol{u})\right), \\
\frac{\partial(\rho E)}{\partial t}+\operatorname{div}(\rho(E \boldsymbol{u}+\boldsymbol{T} \boldsymbol{u}+\boldsymbol{q}))=\operatorname{div}\left(\frac{D_{\alpha}}{p} \nabla(p E)\right),
\end{gathered}
$$

where $D_{\alpha}$ is the empirically scaled mass diffusivity of the gas [1]. Above, the pressure $p$ and the temperature tensor $\boldsymbol{T}$ are further given by

$$
p=\rho \theta, \quad \boldsymbol{T}=\theta \boldsymbol{I}+\boldsymbol{S}, \quad \theta=(\gamma-1)\left(E-\frac{1}{2}\|\boldsymbol{u}\|^{2}\right),
$$

with $\theta$ and $\gamma$ being the temperature of the gas in energy units and the adiabatic exponent of the gas, respectively. As we can see, the equations in (2.1)-(2.2) are closed under all variables except for the stress $\boldsymbol{S}$ and heat flux $\boldsymbol{q}$.

2.1. The Navier-Stokes closure for the stress and heat flux. In the Navier-Stokes closure of the gas dynamics, the stress $\boldsymbol{S}$ and heat flux $\boldsymbol{q}$ are approximated from the Newton and Fourier laws as follows [1]:

$$
\begin{gathered}
\rho \boldsymbol{S}_{N S}=-\mu\left(\nabla \boldsymbol{u}+(\nabla \boldsymbol{u})^{T}+(1-\gamma)(\operatorname{div} \boldsymbol{u}) \boldsymbol{l}\right), \\
\rho \boldsymbol{q}_{N S}=-\frac{\gamma}{\gamma-1} \frac{\mu}{P r} \nabla \theta .
\end{gathered}
$$

Above, $\mu$ and $\operatorname{Pr}$ are the viscosity and the Prandtl number of the gas, respectively. Together with (2.1) and (2.2), the relations in (2.3) comprise the diffusive Navier-Stokes equations [1]. The conventional Navier-Stokes equations [3] result by setting the scaled mass diffusivity coefficient $D_{\alpha}$ to zero.

2.2. The Grad equations for the stress and heat flux. In the diffusive Grad closure of the gas dynamics, the stress $\boldsymbol{S}$ and heat flux $\boldsymbol{q}$ are endowed with their own transport equations $[1,6,10]$

$$
\begin{aligned}
& \frac{\partial(\rho \boldsymbol{S})}{\partial t}+\operatorname{div}(\rho(\boldsymbol{u} \otimes \boldsymbol{S}+\boldsymbol{Q}))+\left(\boldsymbol{P}+\boldsymbol{P}^{T}+(1-\gamma) \operatorname{tr}(\boldsymbol{P}) \boldsymbol{l}\right)= \\
&=-\frac{\rho p}{\mu} \boldsymbol{S}+\operatorname{div}\left(\frac{D_{\alpha}}{p} \nabla(p \boldsymbol{S})\right)
\end{aligned}
$$




$$
\begin{aligned}
\frac{\partial(\rho \boldsymbol{q})}{\partial t}+\operatorname{div}(\rho \boldsymbol{u} \boldsymbol{q})+\operatorname{div}\left(\boldsymbol{P}_{2} \boldsymbol{T}_{2}\right)-\boldsymbol{T}_{2} \operatorname{div} \boldsymbol{P}_{2}-\operatorname{div}\left(\rho \boldsymbol{S}^{2}\right)+ \\
+\rho(\nabla \boldsymbol{u})^{T} \boldsymbol{q}+\frac{\gamma-1}{\gamma} \rho\left[\nabla \boldsymbol{u}+(\nabla \boldsymbol{u})^{T}+(\operatorname{div} \boldsymbol{u}) \boldsymbol{I}\right] \boldsymbol{q}+ \\
\quad+\rho \boldsymbol{Q}:(\nabla \boldsymbol{u})+\operatorname{div}(\rho \boldsymbol{R})=-\operatorname{Pr} \frac{\rho^{2} \theta}{\mu} \boldsymbol{q}+\operatorname{div}\left(\frac{D_{\alpha}}{p} \nabla(p \boldsymbol{q})\right)
\end{aligned}
$$

where

$$
\begin{gathered}
\boldsymbol{P}=\left(\rho \boldsymbol{T}-D_{\alpha}(\nabla \boldsymbol{u})^{T}\right) \nabla \boldsymbol{u}+\frac{\gamma-1}{\gamma} \nabla(\rho \boldsymbol{q}), \\
\boldsymbol{T}_{2}=\boldsymbol{T}+\frac{\theta}{\gamma-1} \boldsymbol{I}, \quad \boldsymbol{P}_{2}=\rho \boldsymbol{T}-2 D_{\alpha} \nabla \otimes \boldsymbol{u} .
\end{gathered}
$$

Observe that the equations in (2.1) and (2.4) are not closed with respect to the matrix $\boldsymbol{R}$ and the 3-rank tensor $\boldsymbol{Q}$ above. For the classical Grad closure [6,7], both $\boldsymbol{Q}$ and $\boldsymbol{R}$ are set to zero. For the regularized Grad closure [1,12,13,15], $\boldsymbol{Q}$ and $\boldsymbol{R}$ are set to

$$
\begin{gathered}
\boldsymbol{Q}=\widetilde{\boldsymbol{Q}}+\widetilde{\boldsymbol{Q}}^{T}+\widetilde{\boldsymbol{Q}}^{T T}, \\
\boldsymbol{R}=\widetilde{\boldsymbol{R}}+\widetilde{\boldsymbol{R}}^{T}+(\widetilde{R}+(1-\gamma) \operatorname{tr}(\widetilde{\boldsymbol{R}})) \boldsymbol{I},
\end{gathered}
$$

where the notations $\widetilde{\boldsymbol{Q}}, \widetilde{R}$ and $\widetilde{\boldsymbol{R}}$ read

$$
\begin{aligned}
& \rho \widetilde{\boldsymbol{Q}}=-\frac{\mu}{P r_{\widetilde{\boldsymbol{Q}}}}\left[\nabla \boldsymbol{S}-\frac{\gamma-1}{\gamma} \boldsymbol{I} \otimes \operatorname{div} \boldsymbol{S}-\frac{1}{p}\left(\boldsymbol{S} \otimes \operatorname{div}(\rho \boldsymbol{S})-\frac{\gamma-1}{\gamma} \boldsymbol{I} \otimes \boldsymbol{S} \operatorname{div}(\rho \boldsymbol{S})\right)+\right. \\
& \left.+\frac{\gamma-1}{\gamma \theta}\left(\boldsymbol{q} \otimes\left(\nabla \boldsymbol{u}+(\nabla \boldsymbol{u})^{T}\right)-\frac{\gamma-1}{\gamma} \boldsymbol{I} \otimes\left(\nabla \boldsymbol{u}+(\nabla \boldsymbol{u})^{T}+(\operatorname{div} \boldsymbol{u}) \boldsymbol{l}\right) \boldsymbol{q}\right)\right], \\
& \rho \widetilde{R}=-\frac{2 \mu}{\operatorname{Pr}_{\widetilde{R}}}\left[\frac{\gamma}{\theta} \operatorname{div}(\theta \boldsymbol{q})-\operatorname{div} \boldsymbol{q}+(\gamma-1)\left(\boldsymbol{S}:(\nabla \boldsymbol{u})-\frac{1}{p} \boldsymbol{q}^{T} \operatorname{div}(\rho \boldsymbol{S})\right)\right], \\
& \text { (2.7c) } \rho \widetilde{\boldsymbol{R}}=-\frac{\mu}{P r_{\widetilde{\boldsymbol{R}}}}\left[\boldsymbol{S}\left(\nabla \boldsymbol{u}+(\nabla \boldsymbol{u})^{T}\right)+\frac{2 \gamma-1}{\gamma \theta}\left(\nabla(\theta \boldsymbol{q})-\frac{1}{\rho} \boldsymbol{q} \operatorname{div}(\rho \boldsymbol{S})^{T}\right)-\right. \\
& \left.-\left((\gamma-1) \operatorname{div} \boldsymbol{u}+\frac{2 \gamma-1}{2 \theta}\left(\frac{1}{\rho} \operatorname{div}(\rho \boldsymbol{q})+\boldsymbol{S}:(\nabla \boldsymbol{u})\right)\right) \boldsymbol{s}\right] .
\end{aligned}
$$

Above, the constants $\operatorname{Pr}_{\widetilde{\boldsymbol{Q}}}, \operatorname{Pr}_{\widetilde{R}}$ and $P r_{\widetilde{\boldsymbol{R}}}$ are the third- and fourth-moment Prandtl numbers, which equal $3 / 2,2 / 3$ and $7 / 6$, respectively, for an ideal monatomic gas $[12,13,15]$. 
2.3. Near-wall scaling of viscosity and mass diffusivity. Previously in [1,2], it was found that, due to the shrinkage of the molecular mean free path in the vicinity of a wall, the viscosity and mass diffusivity should be scaled near channel walls as

$$
\frac{\mu^{\text {near wall }}}{\mu}=\frac{D_{\alpha}^{\text {near wall }}}{D_{\alpha}}=1+\frac{1}{2}\left(\frac{x}{\lambda} E_{1}(x / \lambda)-e^{-x / \lambda}\right),
$$

where $x$ is the distance to the wall, $\lambda$ is the standard length of the mean free path away from the wall, and $E_{1}(x)$ is the exponential integral:

$$
E_{1}(x)=\int_{x}^{\infty} \frac{e^{-y}}{y} \mathrm{~d} y .
$$

In the current work, we use the viscosity and mass diffusivity scaling above for all studied closures. We also compare the results against the conventional Navier-Stokes closure without the viscosity scaling. As in [1], for the computation of $E_{1}(x)$ we use the approximation proposed in [14]. To estimate the mean free path $\lambda$ from the thermodynamic quantities, we use the approximate formula given in [5], Chapter 5, eq. (1.3):

$$
\lambda=\frac{\mu}{p} \sqrt{\frac{\pi \theta}{2}} .
$$

\section{Poiseuille microflow}

3.1. The DSMC set-up. The Direct Simulation Monte Carlo (DSMC) method [4] models a gas flow in a direct fashion by computing the motion and collisions of the actual gas molecules. Previously, we used the DSMC simulations in $[1,2]$ to model the Couette flow in a microchannel. For the DSMC simulation of the Poiseuille flow in the present work we used the dsmcFoam ${ }^{1}$ implementation [11] of the DSMC method. We modified the dsmcFoam software implementations to output the stress and heat flux inside the domain, in addition to the density, velocity and temperature.

Observe that the DSMC method does not simulate the deterministic interaction of realistic gas molecules; on the contrary, the molecular collisions in the DSMC method are modeled similarly to those of the artificial "random gas" in [1] (with the exception that the velocity of colliding molecules is also used in the collision selection algorithm [4]). Because of this similarity between the DSMC collisions and the random gas formulation in [1], the empirically determined mass diffusivity coefficient $D_{\alpha}$ (which we found suitable for the simulations studied below) is likely underestimated versus a realistic gas with deterministic molecular interactions.

For the dsmcFoam computations, we implemented a two-dimensional one micrometer wide and four micrometers long channel, partitioned into uniform rectangular cells, with 100 cells across the channel and 200 cells along the channel (such that the size of each cell is $20 \times 10$ nanometers). The coordinate system was oriented so that the $x$-axis was aligned with the direction of the channel (horizontal direction in the figures below), while the $y$-axis pointed across the channel (vertical direction in the figures below).

\footnotetext{
${ }^{1}$ Part of the OpenFOAM software, http:/ / openfoam.org
} 

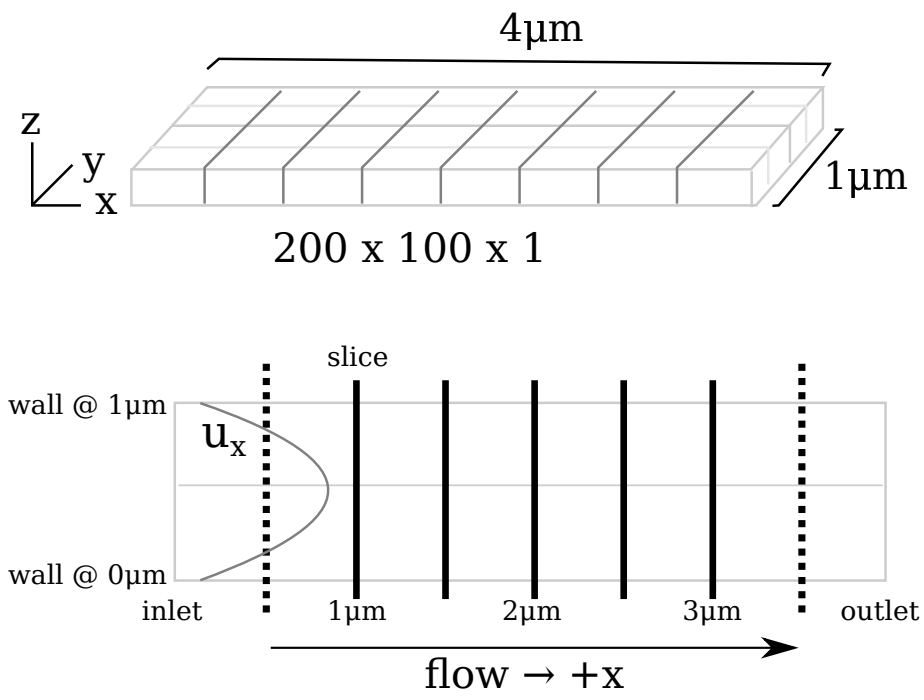

FigurE 1. A schematic representation of the finite volume mesh of the channel and the DSMC set-up for the Poiseuille flow.

At the entrance of the channel, we specified the following thermodynamic parameters for the DSMC simulation:

- The number density and temperature of the incoming gas flow was set to $10^{25}$ molecules per cubic meter and $288.15 \mathrm{~K}\left(15^{\circ} \mathrm{C}\right)$, respectively;

- The velocity of the incoming gas flow was distributed parabolically across the channel, set to 20 meters per second at the walls, and to 100 meters per second in the middle of the channel.

These parameters were used for the simulation of both argon and nitrogen. The resulting pressure was roughly one-half of that at sea level at the entrance of the channel, and onethird of that at the exit. For these conditions, the mean free path of a gas molecule was roughly 150 nanometers long on average, so that the two Knudsen boundary layers (one near each of the two walls) together occupied about one-third of the total width of the channel. To obtain the averaged macroscopic gas flow parameters, we ran the dsmcFoam simulation for $3 \cdot 10^{-5}$ seconds.

Due to the manifestation of computational artifacts in the dsmcFoam simulations immediately adjacent to the entrance and exit of the channel, for the subsequent gas dynamics simulations of the flow we discarded the first and last 0.5 micrometers of the channel (so that the benchmark DSMC flow would be "clean" throughout the remainder of the channel). For this reason, the computational domain of the channel for the gas dynamics simulations below is three micrometers long (instead of four), and starts and ends at 0.5 and 3.5 micrometers, respectively.

For a schematic representation of the finite volume mesh of the channel and the DSMC computational set-up, see Figure 1. 
3.2. The implementation of the continuum gas dynamics methods. We implemented the Navier-Stokes equations (both conventional and diffusive) and the diffusive regularized Grad equations in the OpenFOAM finite volume framework [16]. Due to this reason, for the Navier-Stokes and Grad simulations we imported the same finite volume mesh that was used for the dsmcFoam computations described above. For the diffusive Navier-Stokes and regularized Grad equations, we used the following set of boundary conditions:

- Velocity, temperature, stress and heat flux (the latter two for the Grad equations) the Dirichlet boundary conditions at all boundaries (entrance, exit and the walls), which were imported directly from the dsmcFoam output;

- Pressure - the Dirichlet boundary conditions at the exit and entrance of the channel (imported from the dsmcFoam output), and the Neumann (zero normal gradient) boundary conditions at the walls.

For the conventional Navier-Stokes equations, the set of boundary conditions above is overdetermined, and thus we had to resort to the following "relaxed" set of boundary conditions:

- Velocity - the Dirichlet boundary conditions at the exit and the walls, the Neumann (zero normal gradient) boundary condition at the entrance;

- Temperature - the Dirichlet boundary conditions at all boundaries (entrance, exit and the walls);

- Pressure - the Dirichlet boundary condition at the entrance, the Neumann (zero normal gradient) boundary condition at the exit and the walls.

The reason for this choice of the combination of the Dirichlet and Neumann boundary conditions for the pressure and velocity of the conventional Navier-Stokes equations at the entrance and exit is the qualitative correspondence to the dsmcFoam data; clearly, the pressure gradient at the entrance and the velocity gradient at the exit are nonzeros (see the figures below), and thus we chose to set the Dirichlet boundary conditions for the pressure at the entrance and the velocity at the exit. The corresponding zero-gradient Neumann boundary conditions were then specified at the remaining boundaries.

The following notations are used in all subsequent figures for different types of equations:

- "NS*" - the conventional Navier-Stokes equations (that is, (2.1), (2.2), and (2.3) with $D_{\alpha}=0$ ) without the near-wall viscosity scaling in (2.8);

- "NS" - the conventional Navier-Stokes equations with the near-wall viscosity scaling in (2.8);

- "dNS" - the diffusive Navier-Stokes equations (that is, (2.1), (2.2), and (2.3) with $D_{\alpha}>0$ ) with the near-wall viscosity scaling in (2.8);

- "dRG" - the diffusive regularized Grad equations (that is, (2.1), (2.2), (2.4), (2.6) and (2.7) with $D_{\alpha}>0$ ) with the near-wall viscosity scaling in (2.8);

- "dsmc" - the dsmcFoam simulation.

3.3. Argon. Argon is a monatomic gas, which is often used in computational gas dynamics benchmarks due to its nearly ideal properties. We used the following computational parameters for argon: 


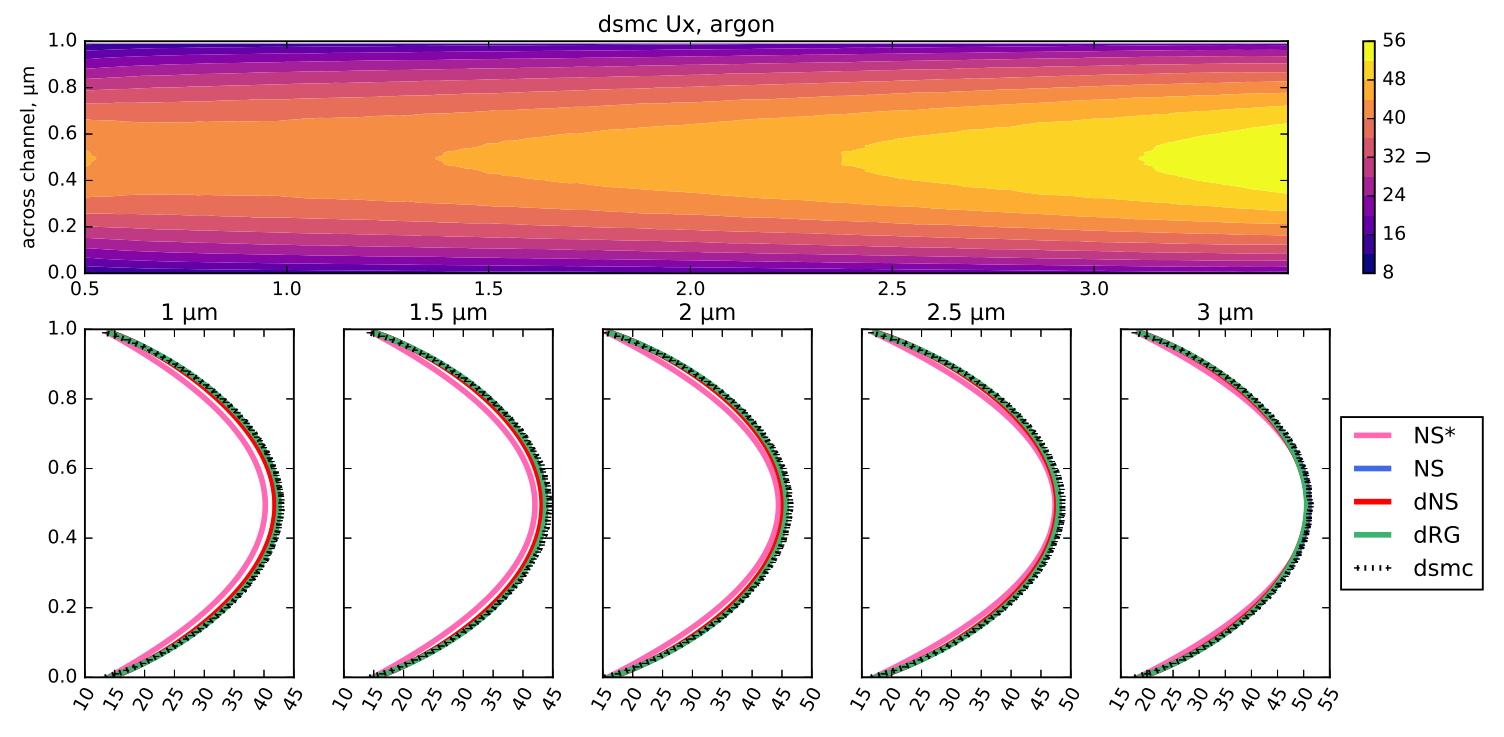

FIGURE 2. Velocity of the Poiseuille flow of $\operatorname{argon}(\mathrm{m} / \mathrm{s})$.

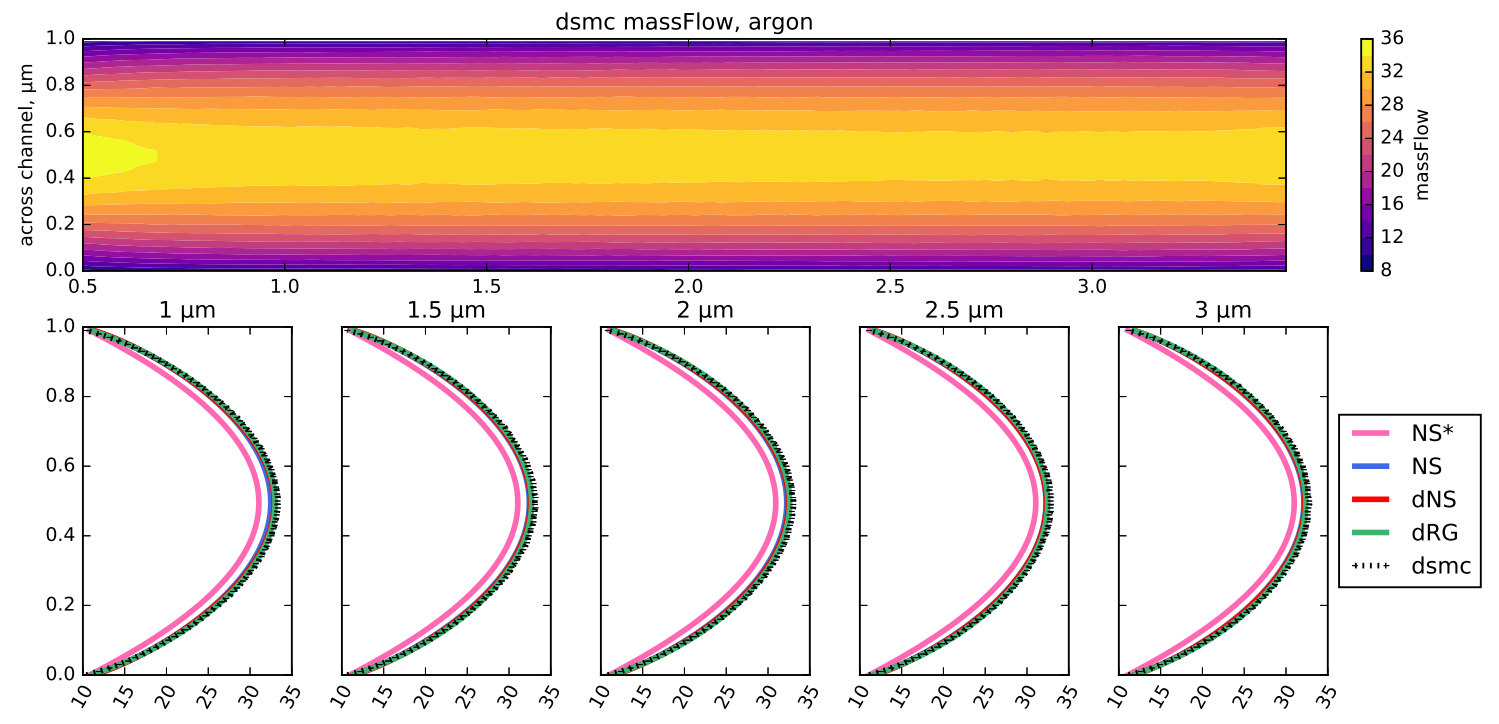

Figure 3. Mass flow of the Poiseuille flow of $\operatorname{argon}\left(\mathrm{kg} /\left(\mathrm{m}^{2} \mathrm{~s}\right)\right)$.

- The adiabatic constant was set to $\gamma=5 / 3$,

- The Prandtl number was set to $P r=2 / 3$,

- The higher-order Prandtl numbers for the regularized Grad equations were set to $P r_{\widetilde{\boldsymbol{Q}}}=3 / 2, \operatorname{Pr}_{\widetilde{R}}=2 / 3$ and $\operatorname{Pr}_{\widetilde{\boldsymbol{R}}}=7 / 6$ (for more details, see $[12,13,15]$ ), 

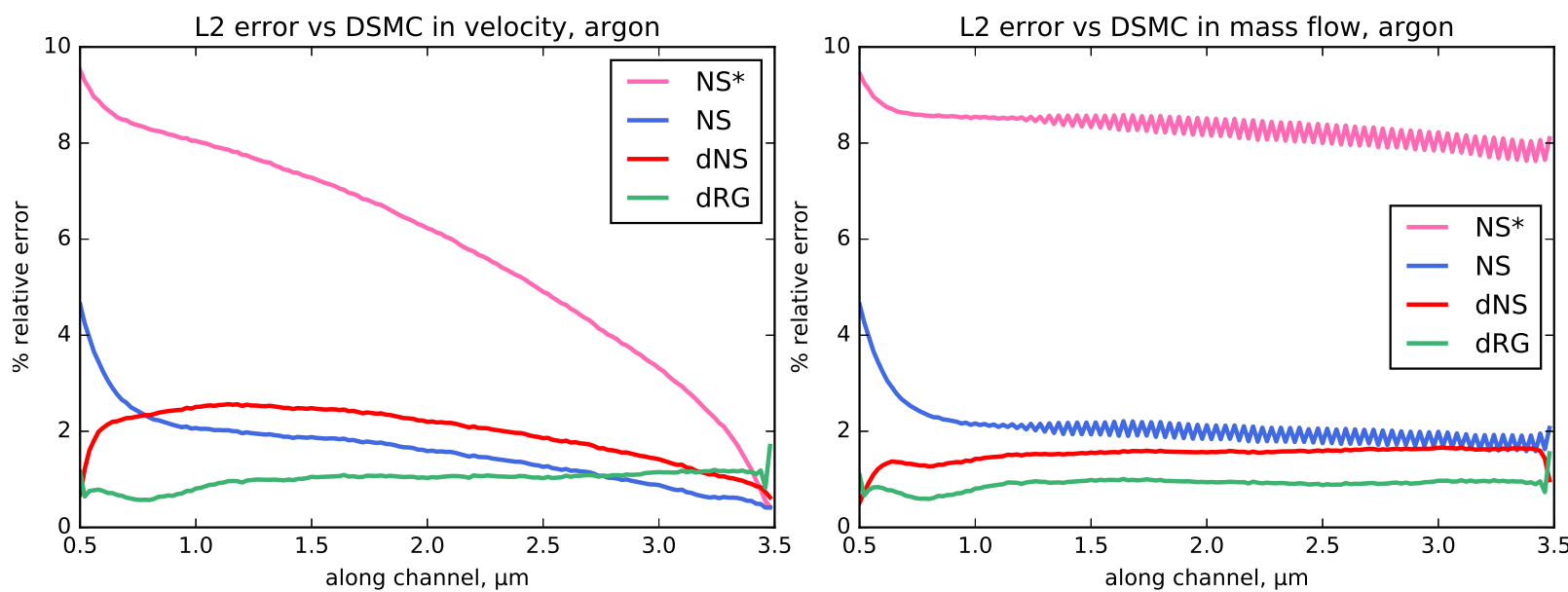

Figure 4. Poiseuille flow of argon, errors in velocity and mass flow.

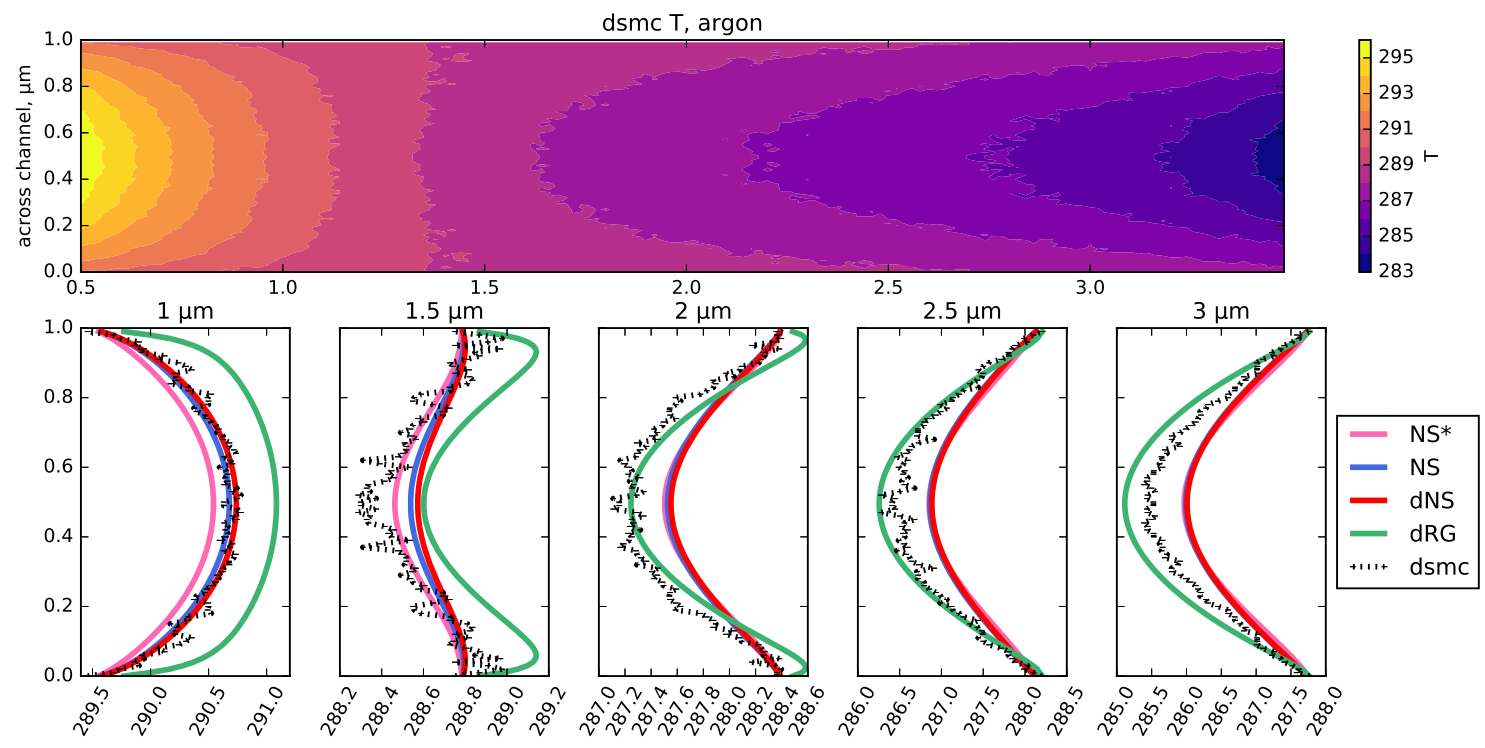

Figure 5. Temperature of the Poiseuille flow of argon (K).

- The molar mass $M$ was set to $3.995 \cdot 10^{-2} \mathrm{~kg} / \mathrm{mol}$,

- The viscosity $\mu$ and empirically scaled mass diffusivity $D_{\alpha}$ were proportional to the square root of the temperature:

$$
\mu=\mu^{*} \sqrt{\frac{M \theta}{R T^{*}}}, \quad D_{\alpha}=D_{\alpha}^{*} \sqrt{\frac{M \theta}{R T^{*}}},
$$

with the reference temperature set to $T^{*}=288.15 \mathrm{~K}$, whereas the reference viscosity and empirical scaled mass diffusivity constants were set to $\mu^{*}=2.2 \cdot 10^{-5}$ 


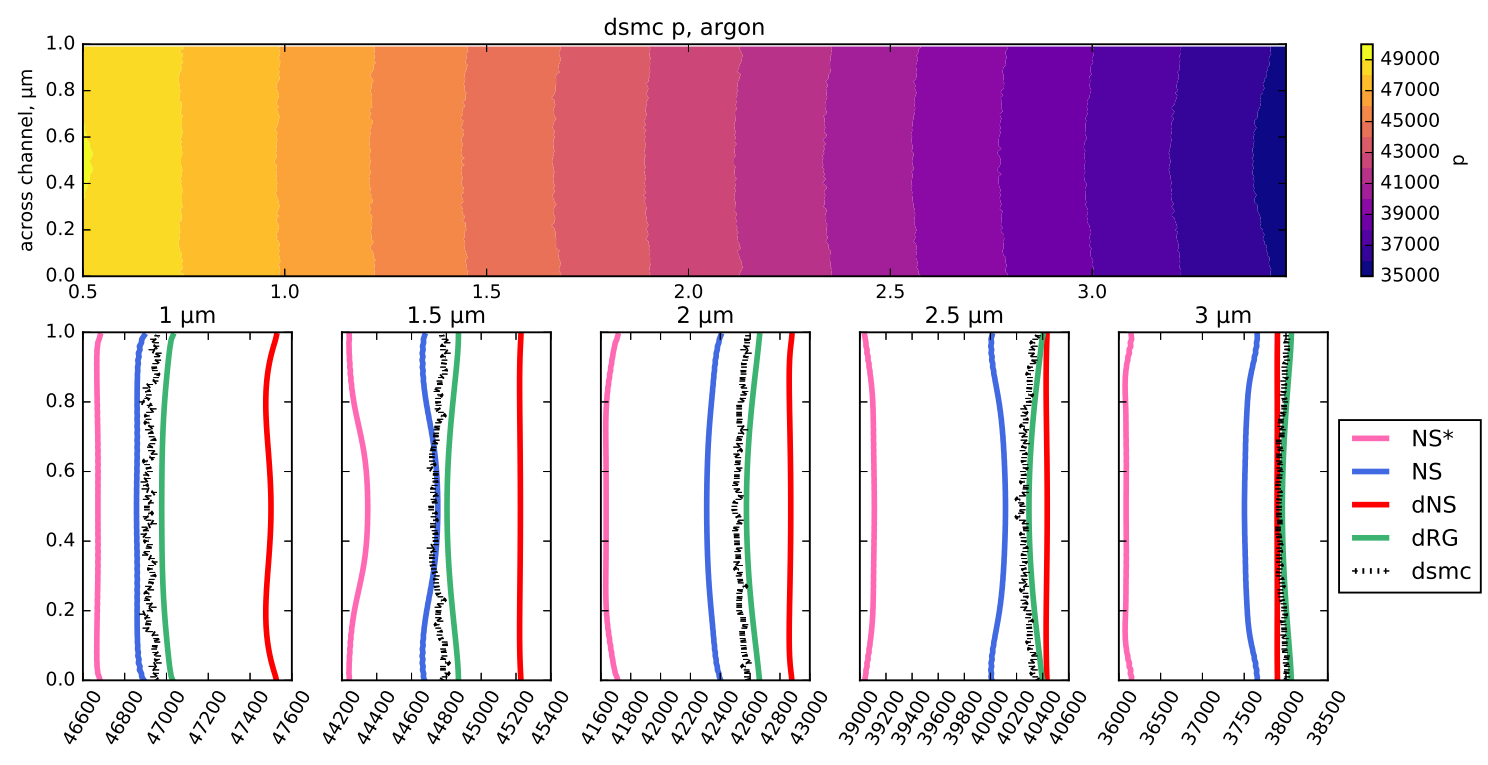

Figure 6. Pressure of the Poiseuille flow of $\operatorname{argon}\left(\mathrm{kg} /\left(\mathrm{m} \mathrm{s}^{2}\right)\right)$.
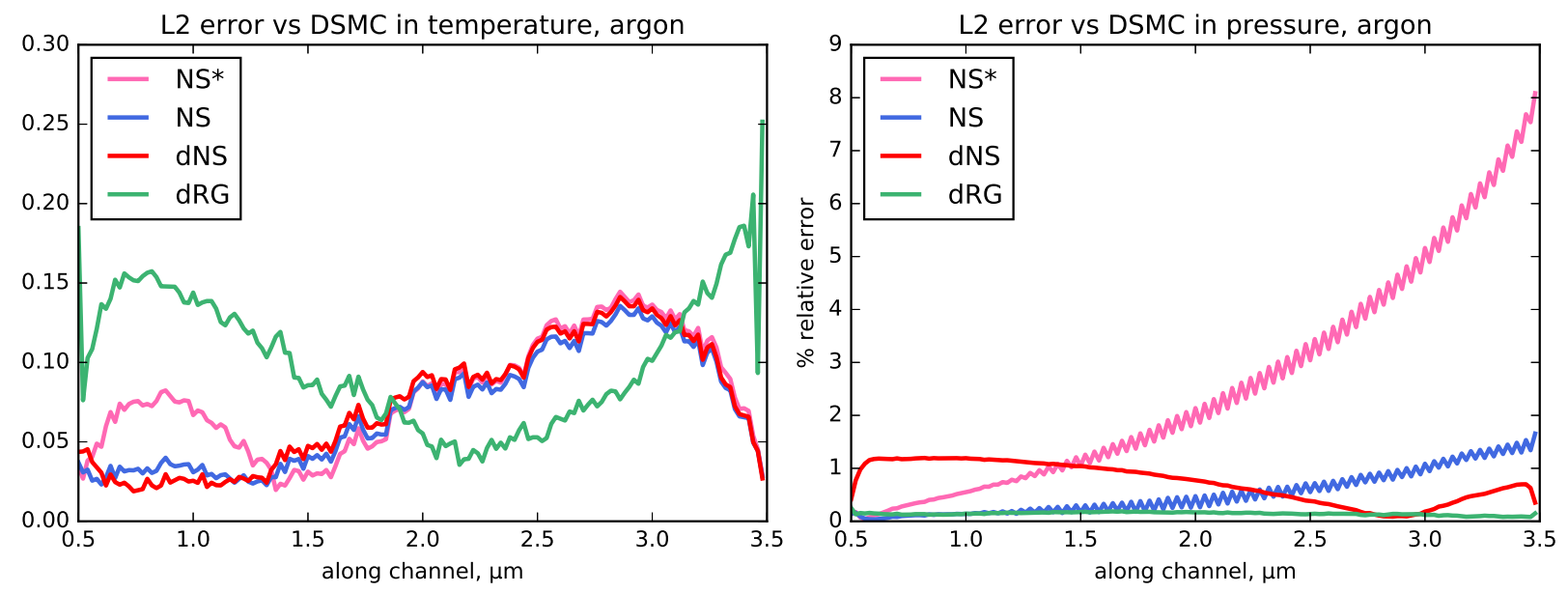

FIGURE 7. Poiseuille flow of argon, errors in temperature and pressure.

$\mathrm{kg} /(\mathrm{m} \mathrm{sec})$, and $D_{\alpha}^{*}=10^{-6} \mathrm{~kg} /(\mathrm{m} \mathrm{sec})$, respectively. The notation $R$ above refers to the universal gas constant, $\left.R=8.314 \mathrm{~kg} \mathrm{~m}^{2} /(\mathrm{mol} \mathrm{K} \mathrm{sec})^{2}\right)$.

In Figures 2-7 we show the velocity, mass flow (the product of the density with velocity), temperature and pressure for the continuum gas dynamics closures and compare them against the DSMC computation. For each variable, we show five evenly spaced profiles across the channel for all studied closures, as well as the corresponding relative errors in across-channel profiles as functions of the distance along the channel. Observe that the conventional Navier-Stokes closure without the viscosity scaling in (2.8) is consistently 


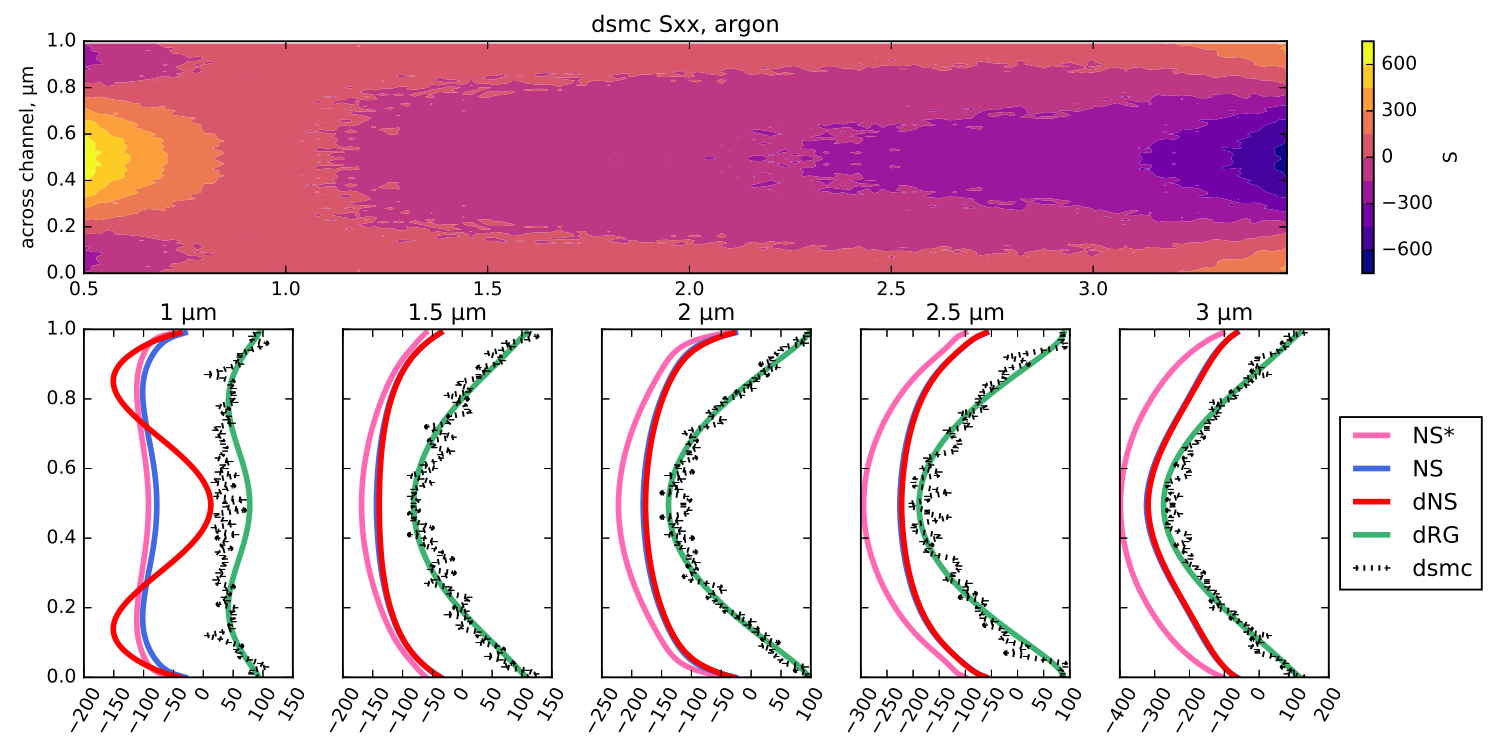

FiguRE 8. Stress component $S_{x x}$ of the Poiseuille flow of $\operatorname{argon}\left(\mathrm{m}^{2} / \mathrm{s}^{2}\right)$.

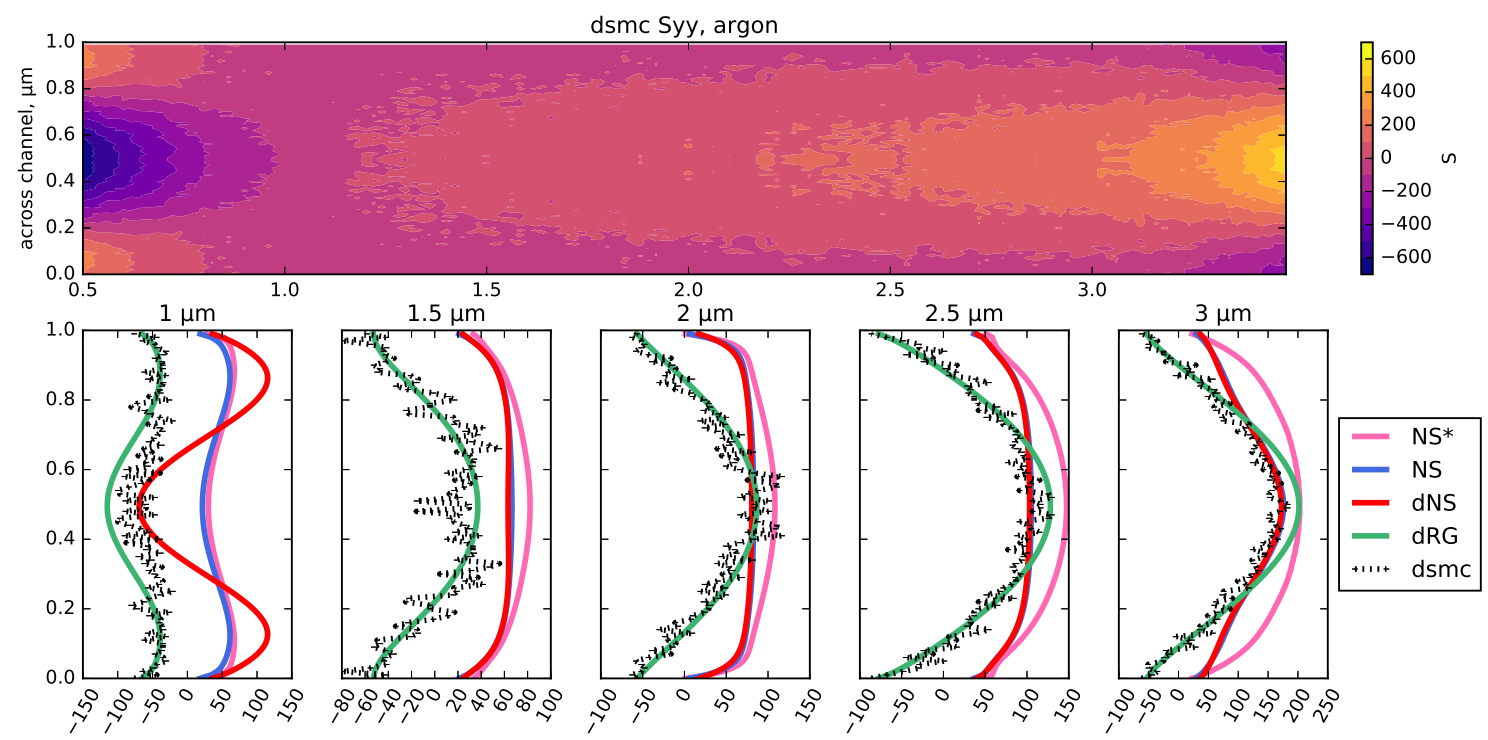

FIGURE 9. Stress component $S_{y y}$ of the Poiseuille flow of $\operatorname{argon}\left(\mathrm{m}^{2} / \mathrm{s}^{2}\right)$.

the least accurate continuum gas dynamics approximation among all tested - its relative error against the DSMC computation reaches $9-10 \%$ in velocity, mass flow and pressure. On the other hand, the diffusive regularized Grad closure with the viscosity scaling in (2.8) is consistently the most accurate continuum gas dynamics closure - its relative 


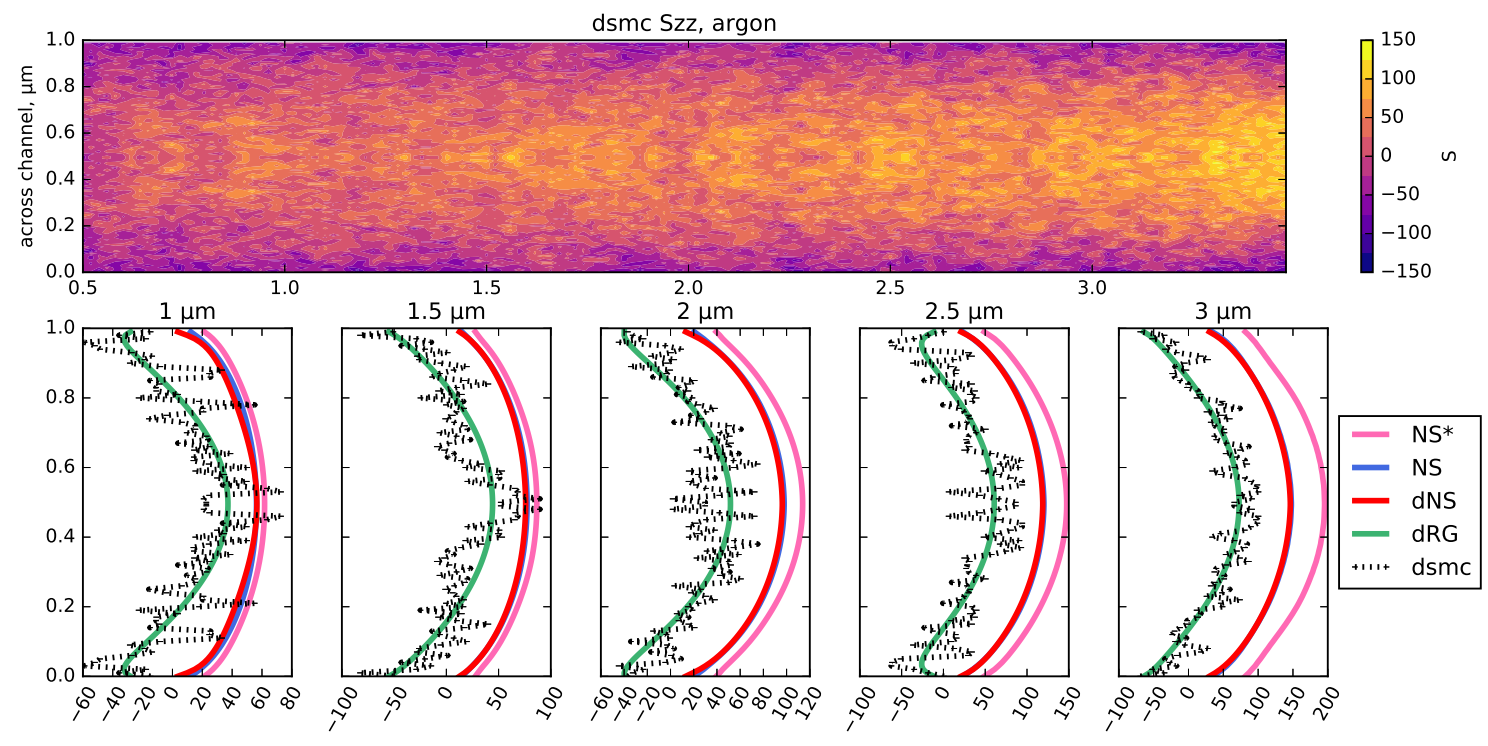

FIgURE 10. Stress component $S_{z z}$ of the Poiseuille flow of argon $\left(\mathrm{m}^{2} / \mathrm{s}^{2}\right)$.

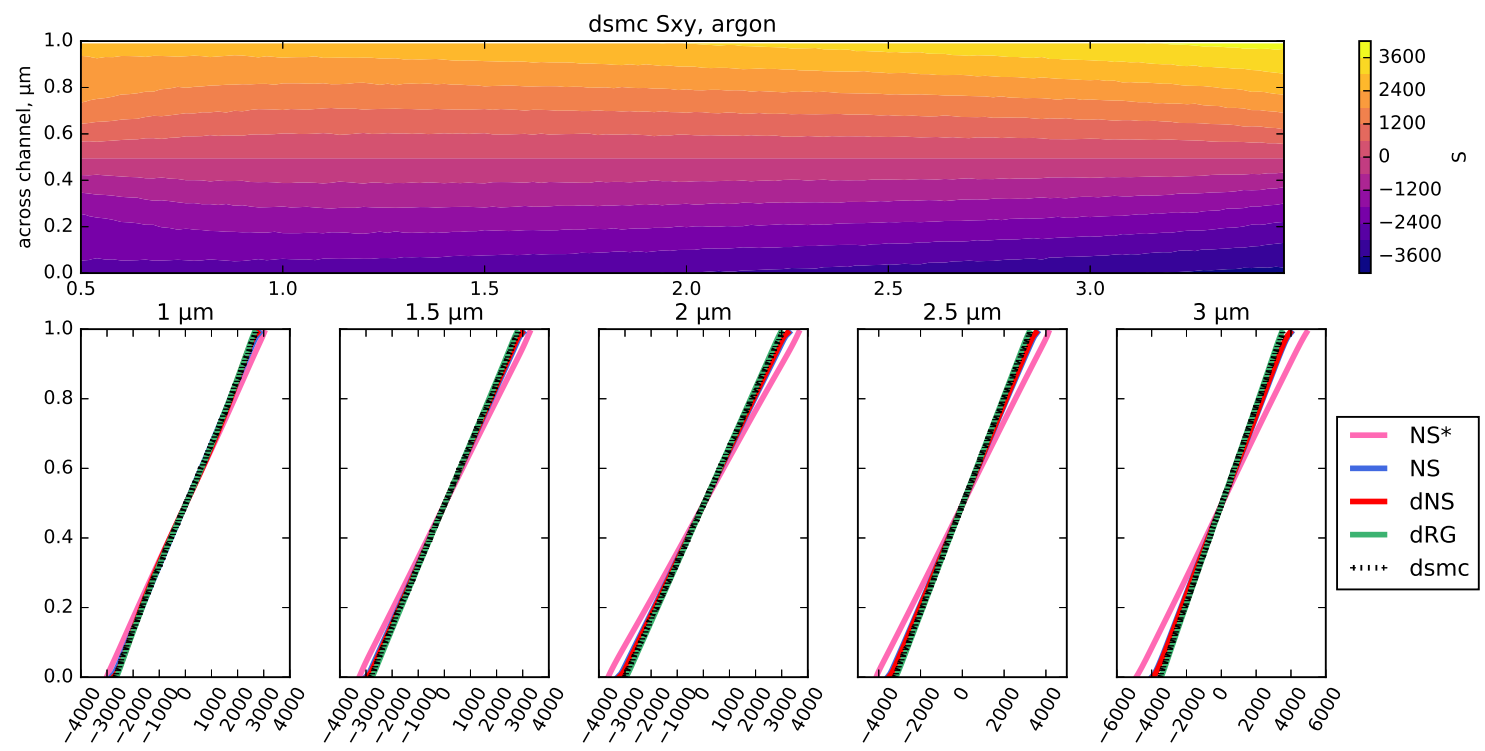

FIGURE 11. Stress component $S_{x y}$ of the Poiseuille flow of argon $\left(\mathrm{m}^{2} / \mathrm{s}^{2}\right)$.

error against the DSMC computation is about $1 \%$ in velocity and mass flow, and about $0.2 \%$ in pressure. The temperature is approximated well by all continuum gas dynamics closures - the errors in temperature are about $0.2 \%$ irrespective of the closure (and is 


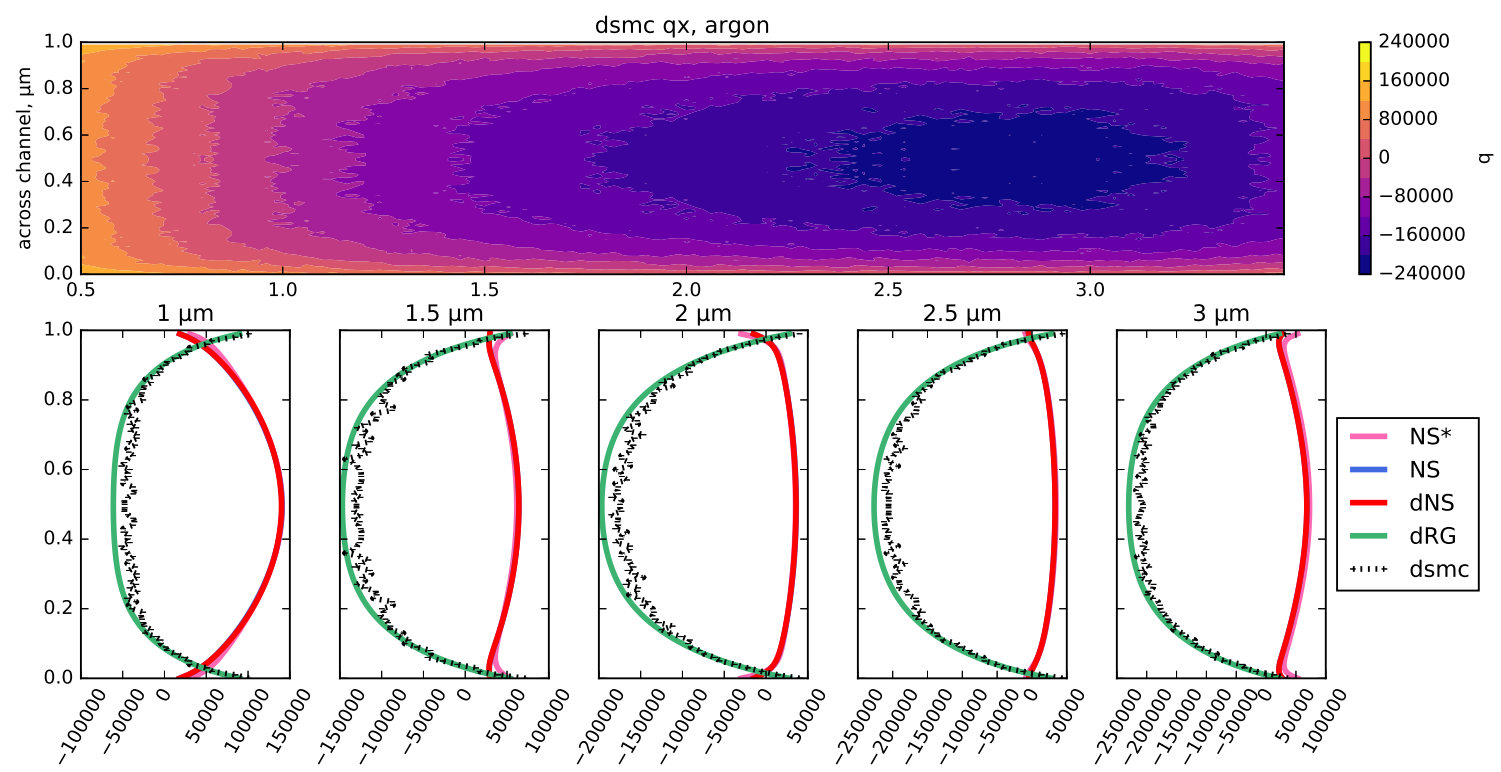

Figure 12. Heat flux component $q_{x}$ of the Poiseuille flow of $\operatorname{argon}\left(\mathrm{m}^{3} / \mathrm{s}^{3}\right)$.

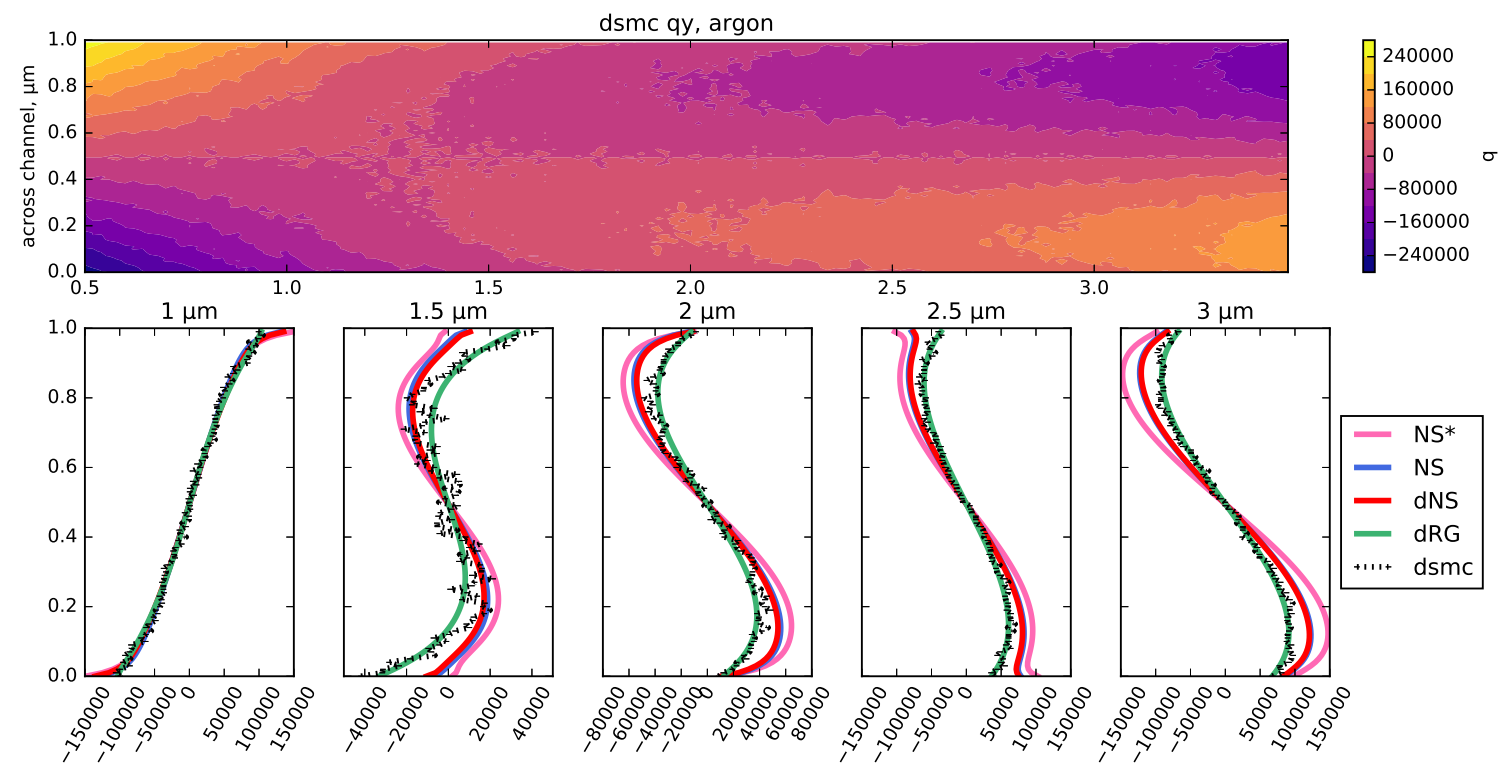

Figure 13. Heat flux component $q_{x}$ of the Poiseuille flow of $\operatorname{argon}\left(\mathrm{m}^{3} / \mathrm{s}^{3}\right)$.

likely due to natural errors of the finite volume approximation of differential operators in general). 


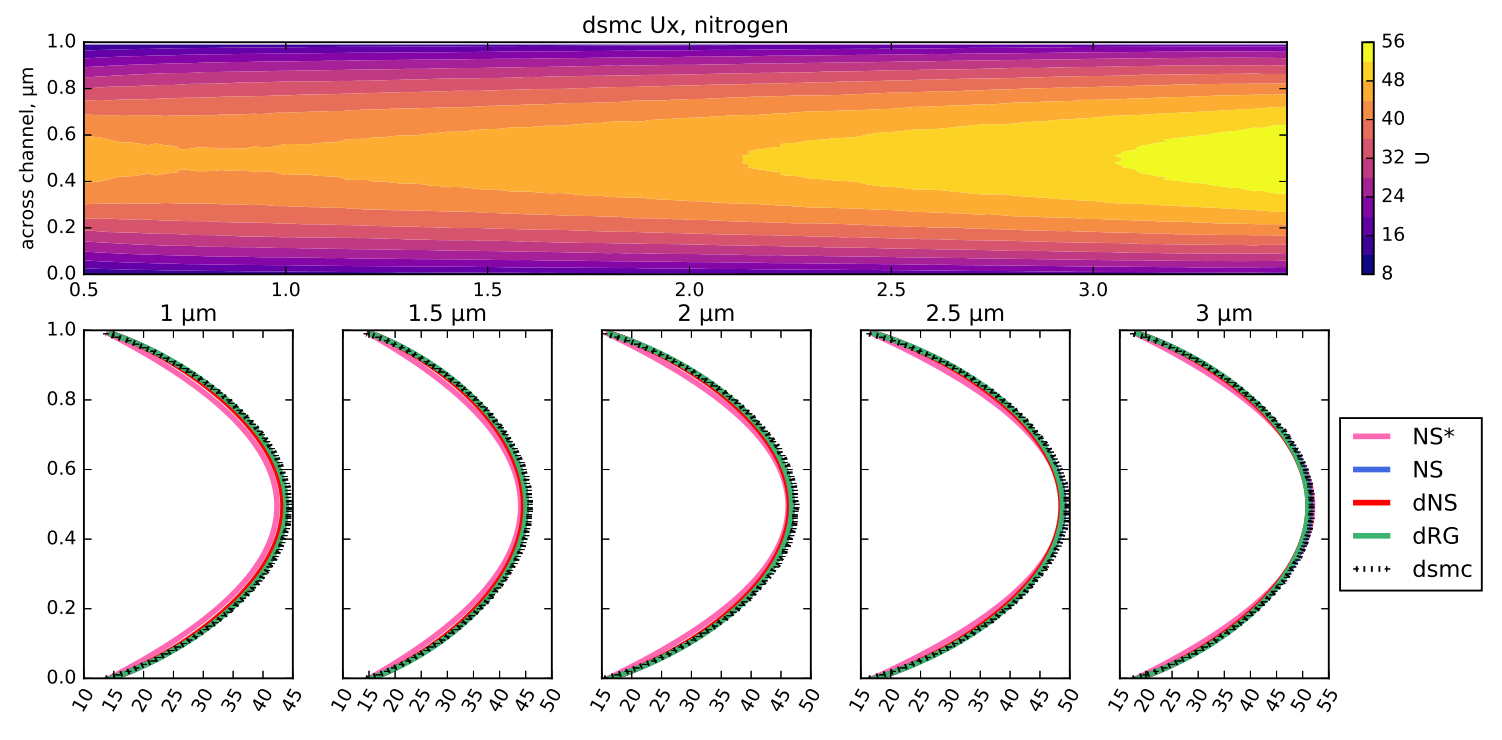

FIgURE 14. Velocity of the Poiseuille flow of nitrogen $(\mathrm{m} / \mathrm{s})$.

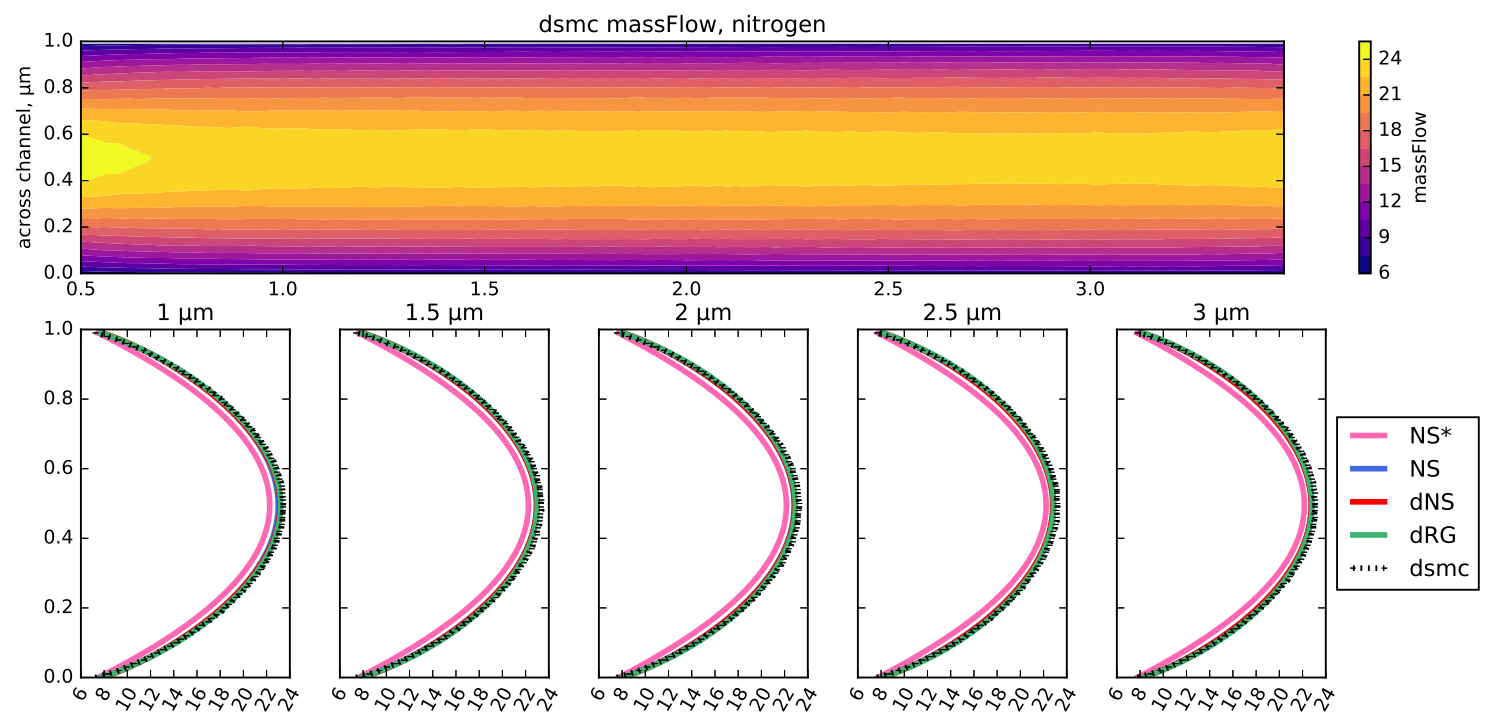

Figure 15. Mass flow of the Poiseuille flow of nitrogen $\left(\mathrm{kg} /\left(\mathrm{m}^{2} \mathrm{~s}\right)\right)$.

Additionally, in Figures 8-13 we show the components of the stress and heat flux for the continuum gas dynamics closures and compare them against the DSMC computation. Observe that the diffusive regularized Grad closure is the only closure which 

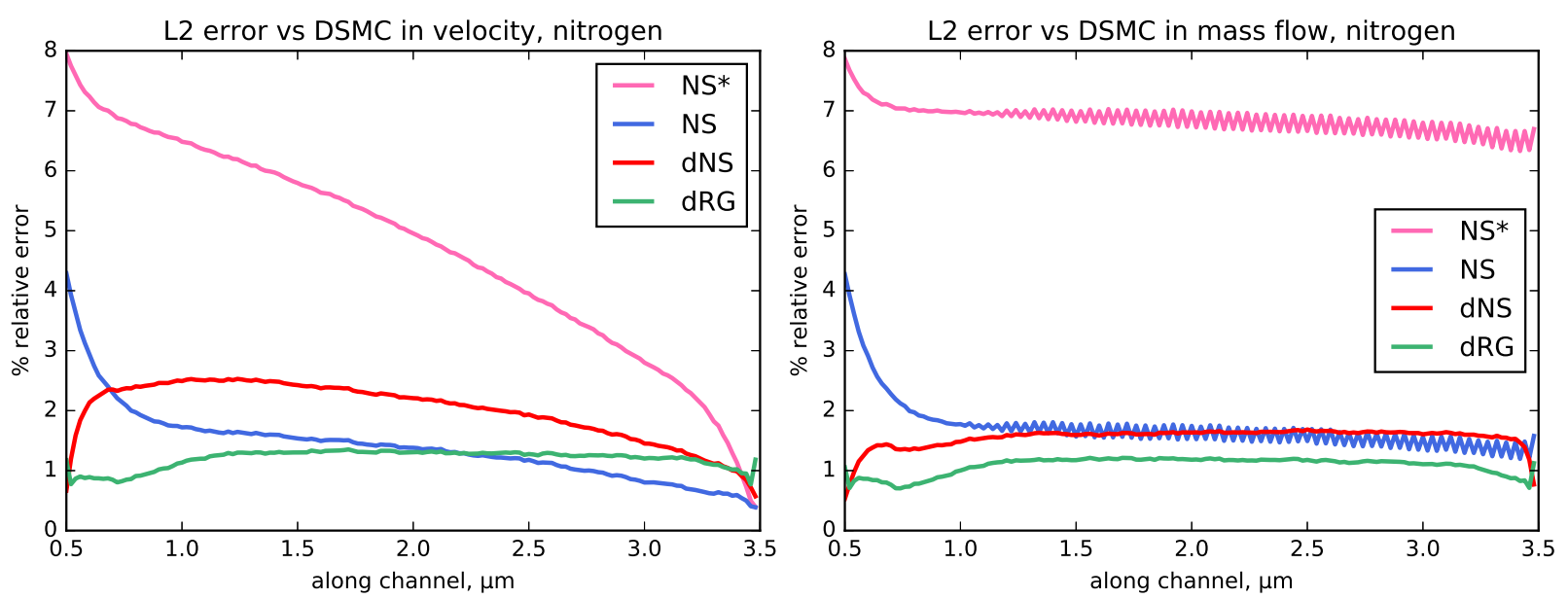

FiguRe 16. Poiseuille flow of nitrogen, errors in velocity and mass flow.

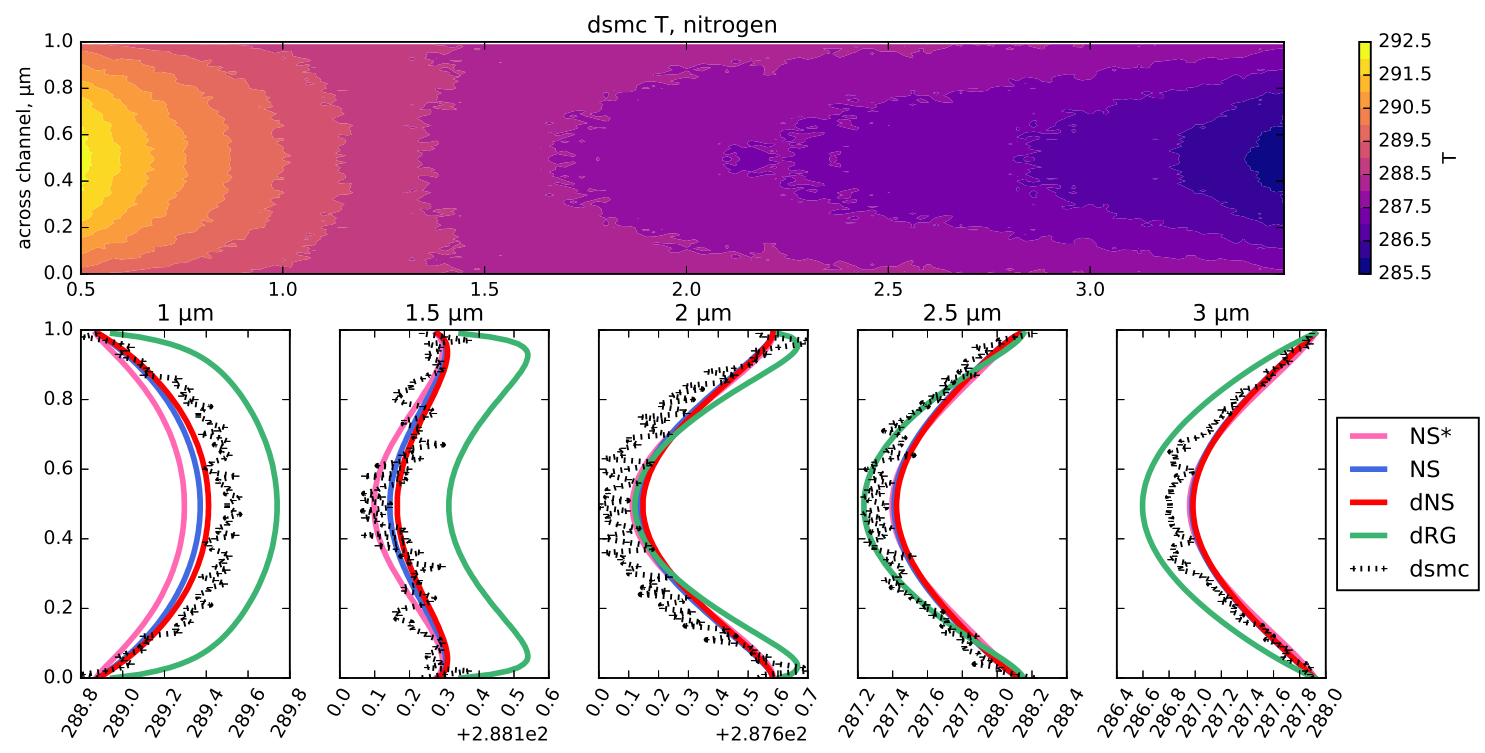

Figure 17. Temperature of the Poiseuille flow of nitrogen (K).

provides moderately accurate approximations of the higher-order moments. The NavierStokes approximations show a good qualitative correspondence for the cross-stress component $S_{x x}$ and the transversal heat flux component $q_{y}$, while all other stress and heat flux components are qualitatively different from the DSMC results.

3.4. Nitrogen. Nitrogen is a diatomic gas and a major component of Earth atmosphere. We used the following computational parameters for argon:

- The adiabatic constant was set to $\gamma=1.4$, 


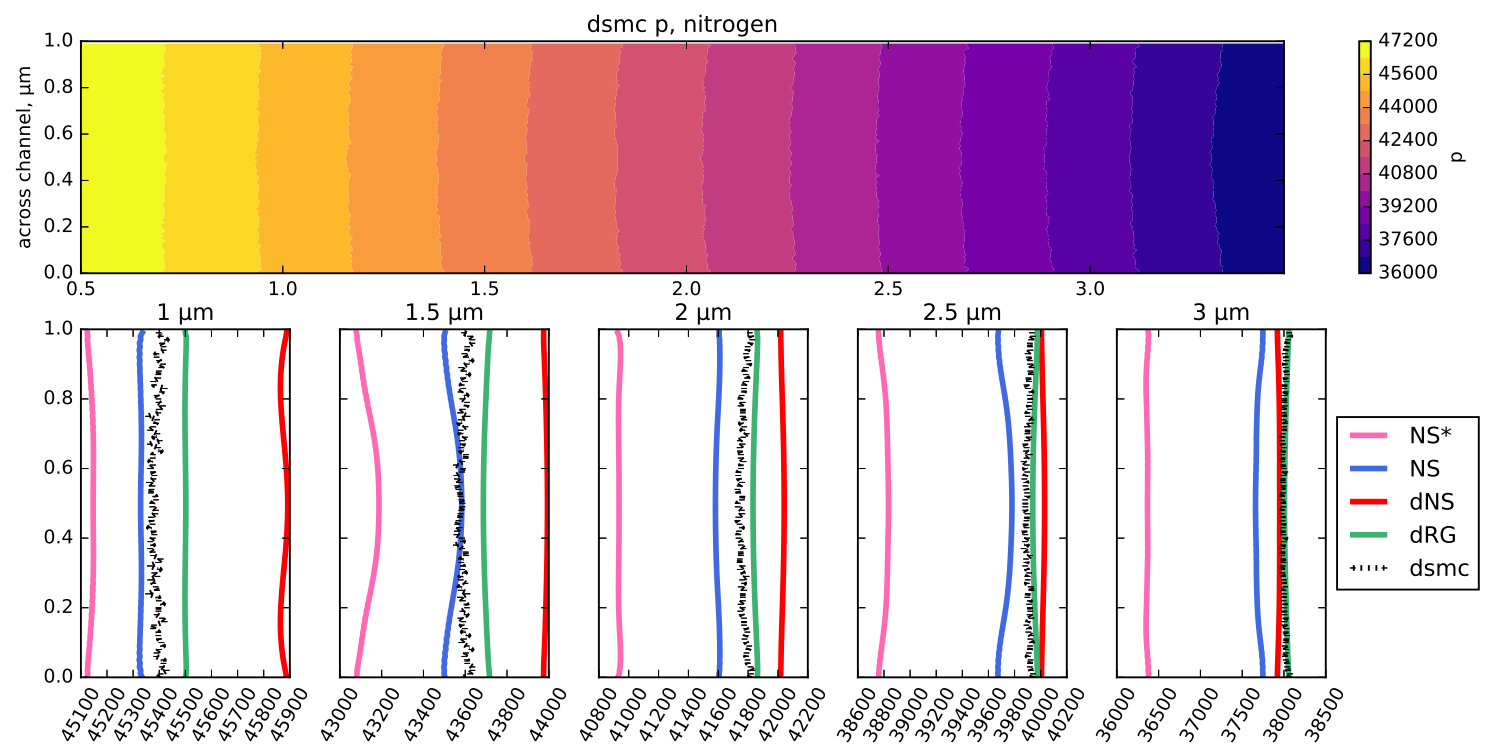

Figure 18. Pressure of the Poiseuille flow of nitrogen $\left(\mathrm{kg} /\left(\mathrm{m} \mathrm{s}^{2}\right)\right)$.
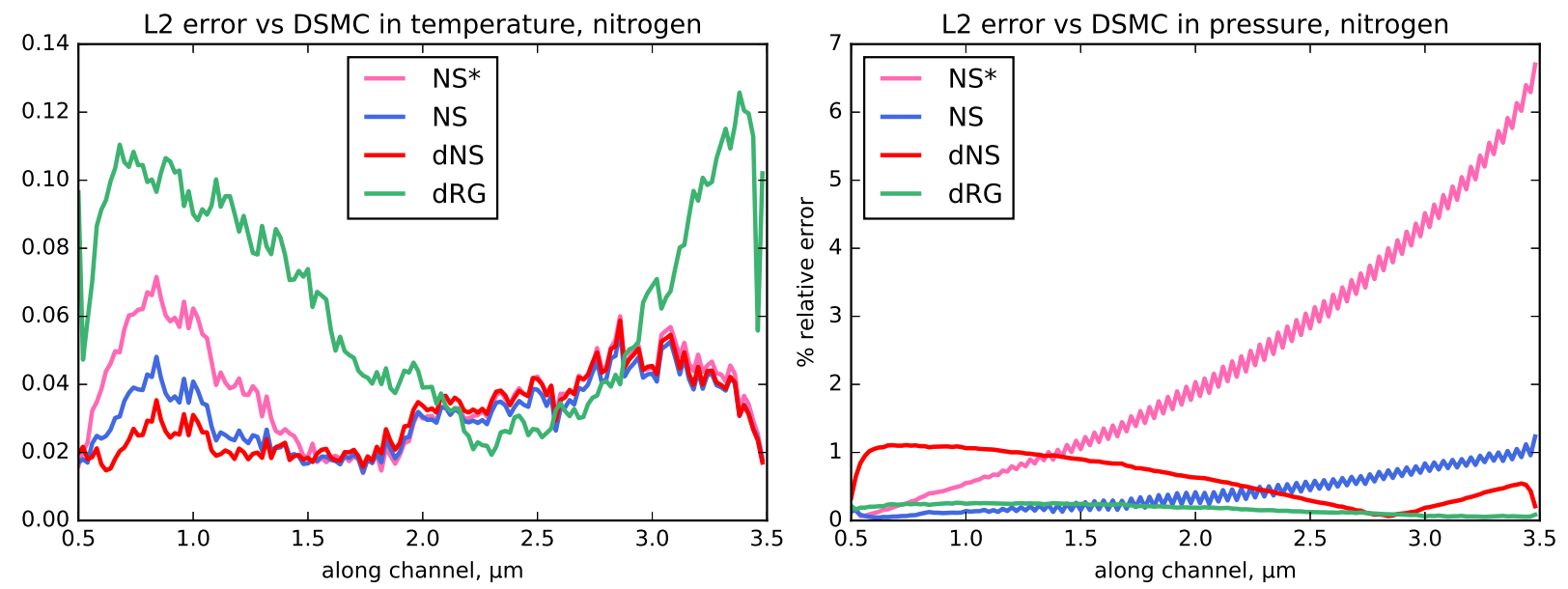

FIGURE 19. Poiseuille flow of nitrogen, errors in temperature and pressure.

- The Prandtl number was set to $P r=0.69$,

- The higher-order Prandtl numbers for the regularized Grad equations were set to the same values as for argon above [1], $P r_{\widetilde{\boldsymbol{Q}}}=3 / 2, P r_{\widetilde{R}}=2 / 3$ and $P r_{\widetilde{\boldsymbol{R}}}=7 / 6$,

- The molar mass $M$ was set to $2.801 \cdot 10^{-2} \mathrm{~kg} / \mathrm{mol}$,

- The viscosity $\mu$ and empirically scaled mass diffusivity were as in (3.1) above, with the reference temperature set to $T^{*}=288.15 \mathrm{~K}$, whereas the reference viscosity and empirical scaled mass diffusivity constants were set to $\mu^{*}=1.74 \cdot 10^{-5} \mathrm{~kg} /(\mathrm{m}$ sec) $[8,9]$, and $D_{\alpha}^{*}=10^{-6} \mathrm{~kg} /(\mathrm{m} \mathrm{sec})$, respectively. 


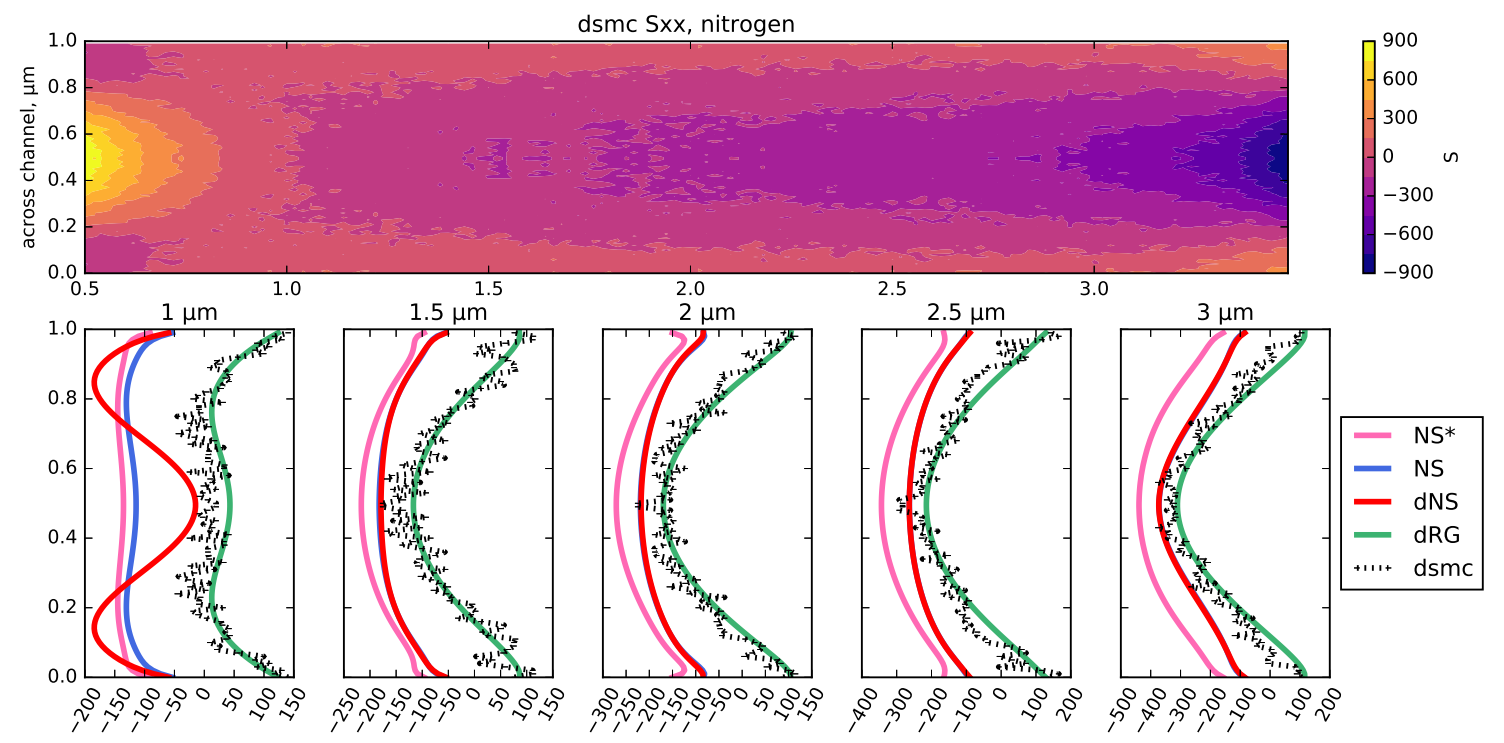

FIgURE 20. Stress component $S_{x x}$ of the Poiseuille flow of nitrogen $\left(\mathrm{m}^{2} / \mathrm{s}^{2}\right)$.

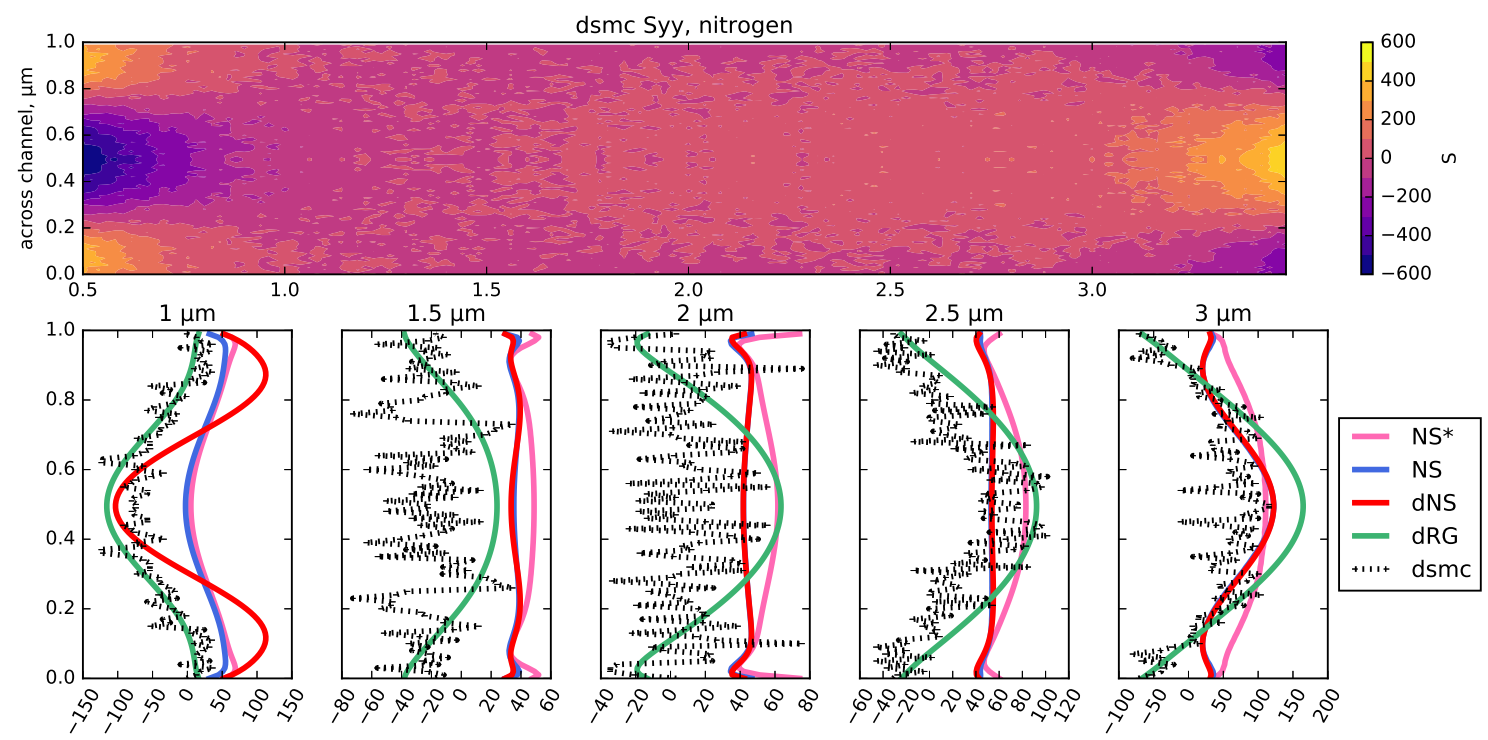

FIgURE 21. Stress component $S_{y y}$ of the Poiseuille flow of nitrogen $\left(\mathrm{m}^{2} / \mathrm{s}^{2}\right)$.

In Figures 14-19 we show the velocity, mass flow (the product of the density with velocity), temperature and pressure for the continuum gas dynamics closures and compare them against the DSMC computation. Just as above for argon, observe that the conventional Navier-Stokes closure without the viscosity scaling in (2.8) is consistently the 


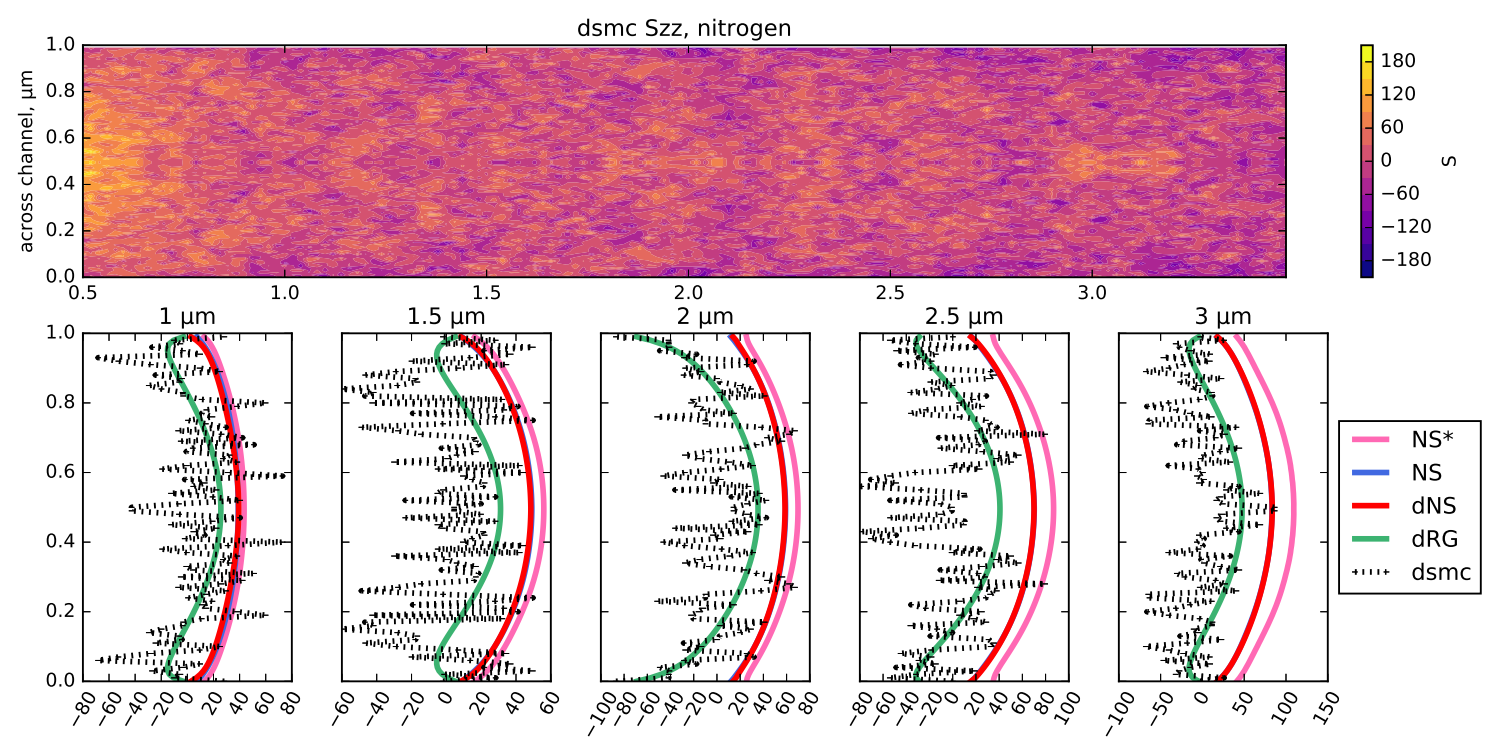

FIgURE 22. Stress component $S_{z z}$ of the Poiseuille flow of nitrogen $\left(\mathrm{m}^{2} / \mathrm{s}^{2}\right)$.

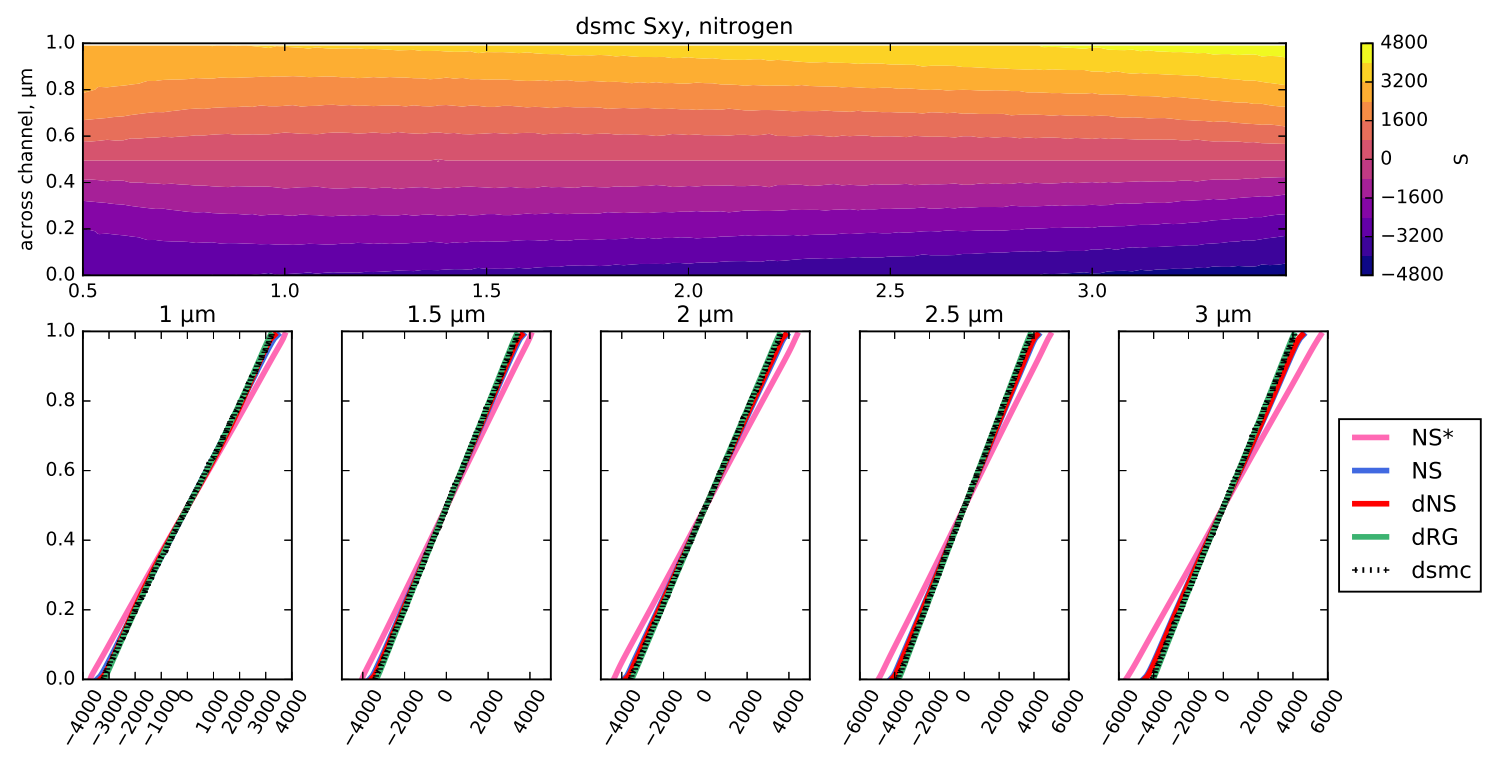

Figure 23. Stress component $S_{x y}$ of the Poiseuille flow of nitrogen $\left(\mathrm{m}^{2} / \mathrm{s}^{2}\right)$.

least accurate continuum gas dynamics approximation for nitrogen among all tested its relative error against the DSMC computation reaches $7-8 \%$ in velocity, mass flow and pressure. On the other hand, the diffusive regularized Grad closure with the viscosity scaling in (2.8) is consistently the most accurate continuum gas dynamics closure - its 


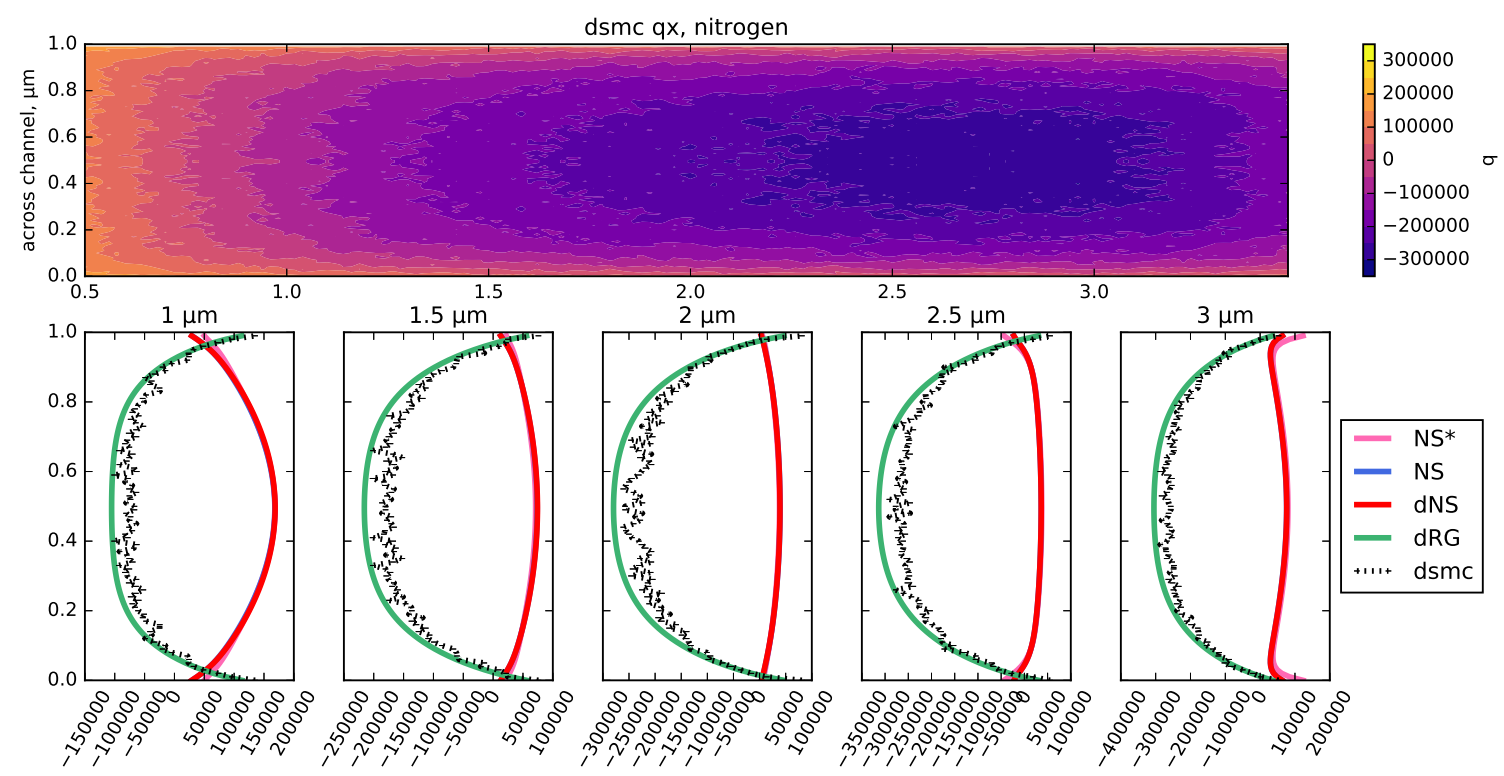

Figure 24. Heat flux component $q_{x}$ of the Poiseuille flow of nitrogen $\left(\mathrm{m}^{3} / \mathrm{s}^{3}\right)$.

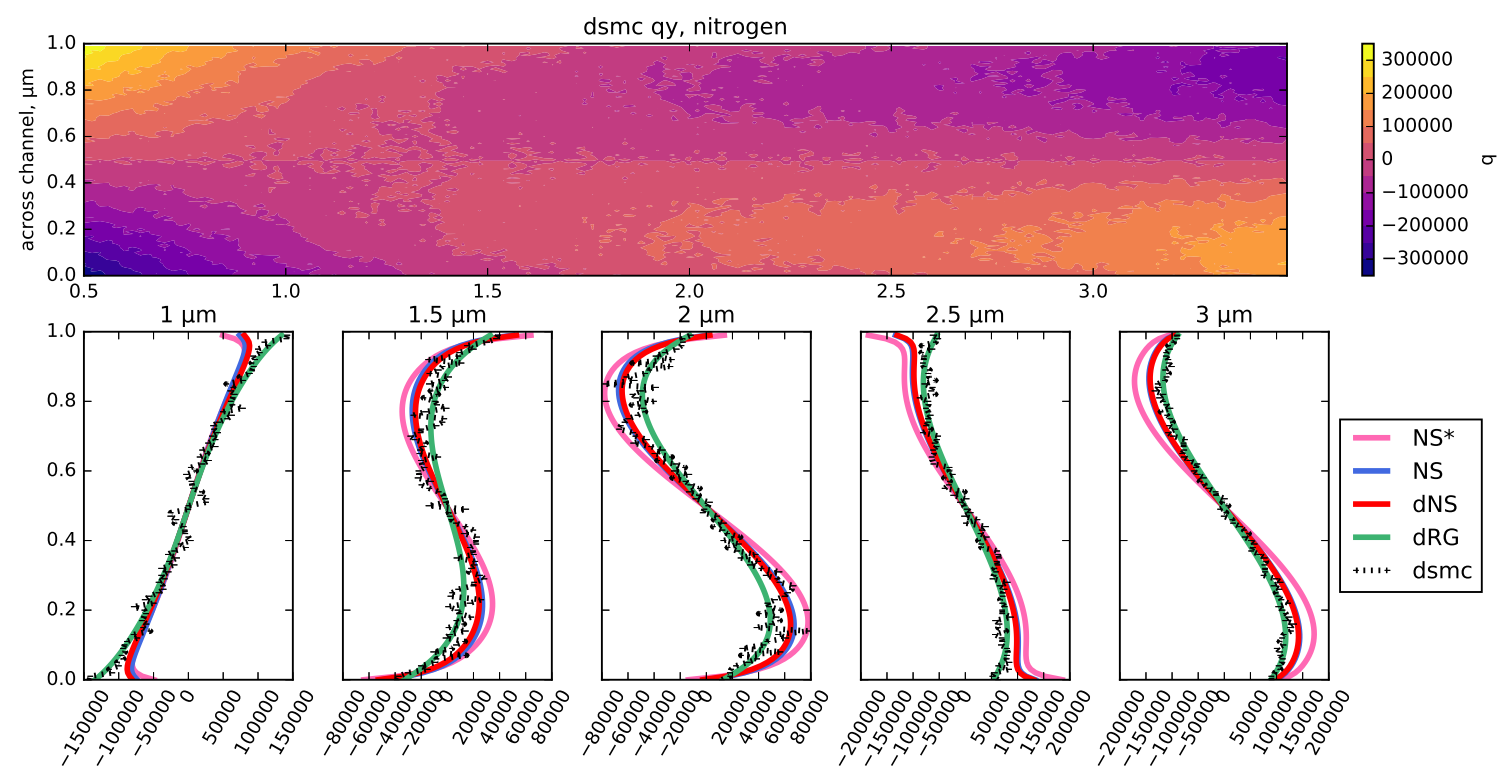

Figure 25. Heat flux component $q_{x}$ of the Poiseuille flow of nitrogen $\left(\mathrm{m}^{3} / \mathrm{s}^{3}\right)$.

relative error against the DSMC computation is about $1 \%$ in velocity and mass flow, and about $0.2 \%$ in pressure. Just as above for argon, the temperature is approximated well by all continuum gas dynamics closures - the errors in temperature are about $0.1 \%$ irrespective of the closure. 
Additionally, in Figures 20-25 we show the components of the stress and heat flux for the continuum gas dynamics closures and compare them against the DSMC computation. Just as above for argon, observe that the diffusive regularized Grad closure is the only continuum gas dynamics closure which provides moderately accurate approximations of the higher-order moments. The Navier-Stokes approximations show a good qualitative correspondence for the cross-stress component $S_{x x}$ and the transversal heat flux component $q_{y}$, while all other stress and heat flux components are qualitatively different from the DSMC results.

\section{SumMARY}

We implement and test the nonequilibrium diffusive gas dynamics equations [1] with the near-wall viscosity scaling [2], considering the Poiseuille microflow in a one micrometer wide channel at close-to-normal thermodynamic conditions (with temperature about $288 \mathrm{~K}$ and pressure between one-half and one-third of that at sea level), simulating both argon (monatomic) and nitrogen (diatomic) gases. By comparison to the statistical DSMC computation, of all the gas dynamics closures tested, the diffusive regularized Grad equations [1] with near-wall viscosity scaling [2] were consistently the most accurate. Its relative errors in thermodynamic variables (i.e. velocity, mass flow, temperature, and pressure) were about $1 \%$ in the worst cases, and we saw qualitatively correct representation of the higher-order moments (stress and heat flux). By contrast, the conventional Navier-Stokes equations without the viscosity scaling were consistently the least accurate (with relative errors up to $10 \%$ ), and the resulting parameterizations for the stress and heat flux qualitatively differed from those recovered by DSMC.

Acknowledgment. The first author was supported by the Office of Naval Research grant N00014-15-1-2036. The second author was supported as a Research Assistant by the same grant.

\section{REFERENCES}

[1] R.V. Abramov. Diffusive Boltzmann equation, its fluid dynamics, Couette flow and Knudsen layers. Physica A, 2017, arXiv:1601.01661. Accepted; DOI 10.1016/j.physa.2017.04.149.

[2] R.V. Abramov. Gas near a wall: a shortened mean free path, reduced viscosity, and the manifestation of a turbulent Knudsen layer in the Navier-Stokes solution of a shear flow. Preprint, 2017, arXiv:1701.02276.

[3] G.K. Batchelor. An Introduction to Fluid Dynamics. Cambridge University Press, New York, 2000.

[4] G.A. Bird. Molecular Gas Dynamics and the Direct Simulation of Gas Flows. Clarendon, Oxford, 1994.

[5] C. Cercignani. The Boltzmann equation and its applications. In Applied Mathematical Sciences, volume 67. Springer, New York, 1988.

[6] H. Grad. On the kinetic theory of rarefied gases. Comm. Pure. Appl. Math., 2(4):331-407, 1949.

[7] H. Grad. Principles of the kinetic theory of gases. In S. Flügge, editor, Handbuch der Physik, volume 12. Springer, Berlin, 1958.

[8] J.O. Hirschfelder, C.F. Curtiss, and R.B. Bird. The Molecular Theory of Gases and Liquids. Wiley, 1964.

[9] E.W. Lemmon and R.T. Jacobsen. Viscosity and thermal conductivity equations for nitrogen, oxygen, argon, and air. Int. J. Thermophys., 25(1), 2004.

[10] F. Mallinger. Generalization of the Grad theory to polyatomic gases. Research Report 3581, Institut National de Recherche en Informatique et en Automatique, 1998. E-print: INRIA-00073100. 
[11] T.J. Scanlon, E. Roohi, C. White, M. Darbandi, and J.M. Reese. An open source, parallel DSMC code for rarefied gas flows in arbitrary geometries. Comput. Fluids, 39(10):2078-2089, 2010.

[12] H. Struchtrup. Grad's moment equations for microscale flows. AIP Conf. Proc., 663:792-799, 2003.

[13] H. Struchtrup and M. Torrilhon. Regularization of Grad's 13-moment equations: Derivation and linear analysis. Phys. Fluids, 15:2668-2680, 2003.

[14] P.K. Swamee and C.S.P. Ojha. Pump test analysis of confined aquifer. J. Irrig. Drain. Eng., 116(1):99$106,1990$.

[15] M. Torrilhon and H. Struchtrup. Regularized 13-moment equations: Shock structure calculations and comparison to Burnett models. J. Fluid Mech., 513:171-198, 2004.

[16] H.G. Weller, G. Tabor, H. Jasak, and C. Fureby. A tensorial approach to computational continuum mechanics using object-oriented techniques. Computers in Physics, 12(6):620-631, 1998. 